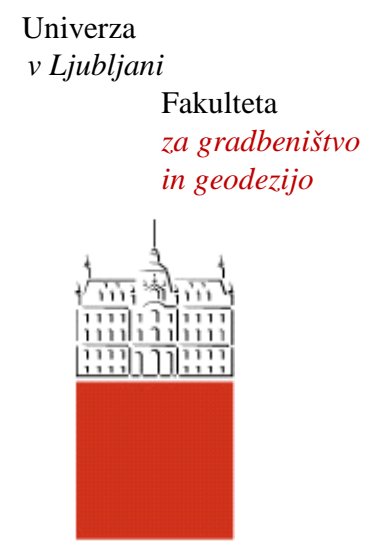

Jamova 2

1000 Ljubljana, Slovenija http://www3.fgg.uni-lj.si//

DRUGG - Digitalni repozitorij UL FGG http://drugg.fgg.uni-lj.si/

Ta članek je avtorjeva zadnja recenzirana različica, kot je bila sprejeta po opravljeni recenziji.

Prosimo, da se pri navajanju sklicujte na bibliografske podatke, kot je navedeno:
University
of Ljubljana

Faculty of

Civil and Geodetic

Engineering

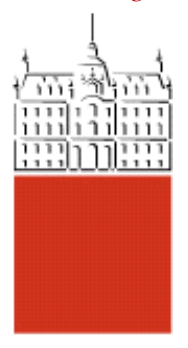

Jamova 2

SI - 1000 Ljubljana, Slovenia

http://www3.fgg.uni-lj.si/

DRUGG - The Digital Repository http://drugg.fgg.uni-lj.si/

This version of the article is author's manuscript as accepted for publishing after the review process.

When citing, please refer to the publisher's bibliographic information as follows:

Bohinc, U., Ibrahimbegović, A., Brank, B. 2009. Model adaptivity for finite element analysis of thin or thick plates based on equilibrated boundary stress resultants. Eng. comput., 26, 1/2, str. 69-99.

DOI: $10.1108 / 02644400910924816$ 


\title{
Model Adaptivity for Finite Element Analysis of Thin or Thick Plates Based on Equilibrated Boundary Stress Resultants
}

\author{
Uroš Bohinc $^{1}$, Adnan Ibrahimbegovic ${ }^{2}$ and Boštjan Brank ${ }^{3}$ \\ ${ }^{1}$ Slovenian Building and Civil Engineering Institute, \\ Ljubljana, Slovenia, e-mail: uros.bohinc@zag.si \\ ${ }^{2}$ Ecole Normale Supérieure, LMT-Cachan, France \\ e-mail: ai@lmt.ens-cachan.fr, fax: +33147402240 \\ ${ }^{3}$ University of Ljubljana, Faculty of Civil and Geodetic Engineering, \\ Ljubljana, Slovenia, e-mail: bbrank@ikpir.fgg.uni-lj.si \\ February 1, 2007
}

\begin{abstract}
Purpose - In this work we address error-controlled adaptive finite element method for thin and thick plates. We present a procedure for determining the most suitable plate model (among available hierarchical plate models) for each particular finite element of the selected mesh, that is provided as the final output of the mesh adaptivity procedure.

Design/methodology/approach - The model adaptivity procedure can be seen as an appropriate extension to model adaptivity for linear elastic plates of so-called equilibrated boundary traction approach error estimates, previously proposed for 2D/3D linear elasticity. Model error indicator is based on a posteriori element-wise computation of improved (continuous) equilibrated boundary stress resultants, and on a set of hierarchical plate models. We illustrate the details of proposed model adaptivity procedure for choosing between two most frequently
\end{abstract}


used plate models: the one of Kirchhoff and the other of ReissnerMindlin. The implementation details are provided for a particular case of the discrete Kirchhoff quadrilateral (DKQ) 4-node plate finite element and the corresponding Reissner-Mindlin quadrilateral (RMQ) with the same number of nodes. The key feature for those elements that they both provide the same quality of the discretization space (and thus the same discretization error) is the one which justifies uncoupling of the proposed model adaptivity from the mesh adaptivity. Findings - Several numerical examples are presented in order to illustrate a very satisfying performance of the proposed methodology in guiding the final choice of the optimal model and mesh in analysis of complex plate structures.

Originality/value - Confirms that one can make an automatic selection of the most appropriate plate model for thin and thick plates on the basis of proposed model adaptivity procedure.

Keywords: plate models, finite elements, model adaptivity, error estimates

Paper type: research paper

\section{Introduction}

Analysis of plate structure with complex shape, loading and boundary conditions is one of the most frequently encountered problems in structural engineering practice. A problem of selecting the most suitable computational model for a particular plate structure, which is the topic of this work, has therefore interesting practical aspects. If successfully solved, it can lead to an efficient and accurate plate analysis, which is of great practical interest. Since plate structures are often combined with frame and other skeletal structures, for which one can develop by far the most efficient finite element analysis by exploiting one dimensional form of the governing model and the superconvergence properties of the corresponding finite element method (e.g. see [29] or [14]), the solution of the above mentioned problem would clearly have a very practical value.

Adaptive modelling in structural analysis has the goal to produce the most suitable computational model for a particular structural component or its part. The computational model suitability is measured in terms of two errors, which normally occur in the finite element modelling: the discretization error and the modelling error. Since adaptive modelling relies on both dis- 
cretization and modelling error, in order to drive the adaptive process of computational modelling, the best possible estimate of both errors is of crucial importance for its success. The discretization error comes as a consequence of a chosen finite element discretization of a particular solid/structural mathematical model. In simple words: it measures how close the discretized finite element solution is to the exact solution of governing equations of the underlying mathematical model. The modelling error is related to the suitability of mathematical model itself. In plate problems it arises because we usually model a plate with simplified two-dimensional (2D) models (i.e. Kirchhoff or Reissner-Mindlin), which are approximations of the three-dimensional (3D) solid model. Among other reasons, such simplifications are of interest due to the computational savings (through dimensional reduction) and higher computational robustness of $2 \mathrm{D}$ plate models with respect to $3 \mathrm{D}$ model in the case of plate problems.

To produce an efficient computational model we thus have to control both the discretization and the modelling error. The discretization error is controlled by suitable meshing of the domain that is usually called mesh or $h$-adaptivity. Many error estimators and corresponding mesh adaptivity procedures are available (see e.g. [4], [22] and [30], and e.g. [18] for plates). On the other hand, not many procedures are suitable for the modelling error control. It has been shown recently e.g. by [25], [21], [26], [23], [27] that modelling error for 2D/3D linear elasticity can be controlled by so-called equilibrated boundary traction approach to error estimation. Our departure point is the assumption that a family of hierarchic plate models is provided, and the adaptive analysis starts with the simplest possible model. From a posteriori computations, that include higher model in the hierarchy suitable model error indicator is obtained. The regions where more refined model should be used can be then identified.

In this work we focus upon the development of model adaptivity procedure for plates. We assume that mesh and model adaptivity can be treated separately; ideally, the model adaptivity procedure should start from the final output of the mesh adaptivity, which would distribute evenly the discretization error throughout the mesh. The presented procedure can be seen as an application of equilibrated boundary traction approach to error estimation to model adaptivity for plates. It can select automatically which particular model (from a set of available hierarchic plate models) is the most suitable for any finite element of the chosen mesh. We address in detail a particular case of two low-order models, i.e Kirchhoff and Reissner-Mindlin ones. We 
note that the procedure developed for these two models (we will call it KRM procedure) has very similar form to the procedure that would include higher hierarchic models, i.e. so-called $(1,1,2),(3,3,4)$ or "zig-zag" plate models that take into account through-the-thickness stretching and nonlinear distribution of displacements through the plate thickness (see e.g. [3] and [9] for those models). The chosen elements are quadrilaterals; i.e discrete Kirchhoff quadrilateral (DKQ) and the corresponding Reissner-Mindlin quadrilateral (RMQ). They have the ability to provide approximately the same order of the discretization error for bending moments, leaving the only difference in the shear part of the error norm, which justifies uncoupling of model and mesh adaptivity.

It is obvious that the main reason for using the model adaptivity procedure that chooses between the DKQ and the RMQ elements is not an increase of efficiency; the DKQ allows only slight computational savings with respect to the RMQ, and one may simply use the RMQ throughout the mesh. However, there are several aspects to such a procedure that deserve attention: (i) as already mentioned above, the KRM procedure can be easily modified to the one that includes higher-order plate models with much more significant savings, (ii) the DKQ elements are robust and very often used as the standard for commercial codes (e.g. SAP2000 [28]) and mesh adaptivity performed with the DKQ elements is easier to handle then by using the RMQ elements (e.g. see [18]), (iii) from the theoretical point of view the KRM procedure can be used to locate regions in plate where shear is of importance.

The outline of the paper is as follows. In Section 2 we present plate finite elements that are used in what follows (see also [13]); they have an exceptional feature of sharing the same order of interpolation for displacement and bending strains. In the same section, we briefly comment on the possible manners of choosing an optimal finite element mesh applicable to any of these models. In Section 3 we discuss how to test any of the plate element of the chosen mesh in order to choose between the Kirchhoff and the Reissner-Mindlin model. In Section 4 we present a number of numerical examples illustrating the proposed procedure satisfying performance. Closing remarks are stated in Section 5. 


\section{Thick and thin plate finite element models}

In this section we briefly present finite elements that will be further used in model adaptivity procedure.

\subsection{Theoretical formulation}

We model a plate as a $2 \mathrm{D}$ body occupying a domain $\Omega$ in the $x_{1} x_{2}$ plane. The weak form of the boundary value problem for the Reissner-Mindlin plate model is given as (e.g. see [6] or [5])

$$
\begin{aligned}
D \Pi\left(w, \theta_{m}\right)\left(\widehat{w}, \widehat{\theta}_{n}\right) & :=\int_{\Omega} \widehat{\kappa}_{i j}\left(\widehat{\theta}_{n}\right) C_{i j k l}^{B} \kappa_{k l}\left(\theta_{m}\right) d \Omega+\int_{\Omega} \widehat{\gamma}_{i}\left(\widehat{w}, \widehat{\theta}_{n}\right) C_{i j}^{S} \gamma_{j}\left(w, \theta_{m}\right) d \Omega \\
& -\int_{\Omega} \widehat{w} f d \Omega=0, \quad i, j, k, l, m, n \in\{1,2\}
\end{aligned}
$$

where $w$ denotes displacement field in $x_{3}$ direction, $\theta_{m}$ is rotation field (of plate normals) in the direction of $x_{m}$ coordinate, $f$ is a surface loading acting on the plate in $x_{3}$ direction, and $(\hat{\cdot})$ is virtual quantity that corresponds to $(\cdot)$. The curvature tensor $\kappa_{i j}$ can be expressed by rotations $\beta_{i}$ as

$$
\kappa_{i j}=\frac{1}{2}\left(\frac{\partial \beta_{i}}{\partial x_{j}}+\frac{\partial \beta_{j}}{\partial x_{i}}\right) ; \quad \beta_{i}=e_{i j} \theta_{j}, e_{i j}=\left[\begin{array}{cc}
0 & -1 \\
+1 & 0
\end{array}\right]
$$

In $(2.1), \gamma_{i}$ are shear strain components

$$
\gamma_{i}=\frac{\partial w}{\partial x_{i}}-\beta_{i}
$$

and $C_{i j k l}^{B}$ and $C_{i j}^{S}$ are components of bending and shear elastic constitutive tensor, respectively.

The Kirchhoff plate model would eliminate shear strains, $\gamma_{i}=0$, thus introducing a new definition of the curvature tensor

$$
\gamma_{i}=0 \Rightarrow \beta_{i}=\frac{\partial w}{\partial x_{i}} \Rightarrow \kappa_{i j}=\frac{\partial^{2} w}{\partial x_{i} \partial x_{j}}
$$

The discrete formulation, which corresponds to (2.1), is

$D \Pi\left(w^{h}, \boldsymbol{\theta}^{h}\right) \cdot\left(\widehat{w}^{h}, \widehat{\boldsymbol{\theta}}^{h}\right):=\int_{\Omega^{h}} \widehat{\boldsymbol{\kappa}}^{h T} \mathbf{C}^{B} \boldsymbol{\kappa}^{h} d \Omega+\int_{\Omega^{h}} \widehat{\boldsymbol{\gamma}}^{h T} \mathbf{C}^{S} \boldsymbol{\gamma}^{h} d \Omega-\int_{\Omega^{h}} \widehat{w}^{h} f d \Omega=0$ 
where the following mappings are defined

$$
\begin{gathered}
\kappa_{i j}^{h} \mapsto \boldsymbol{\kappa}^{h}=\left[-\partial \theta_{2}^{h} / \partial x_{1} ; \partial \theta_{1}^{h} / \partial x_{2} ; \partial \theta_{1}^{h} / \partial x_{1}-\partial \theta_{2}^{h} / \partial x_{2}\right]^{T} \\
\gamma_{i}^{h} \mapsto \gamma^{h}=\left[\partial w^{h} / \partial x_{1}+\theta_{2}^{h} ; \partial w^{h} / \partial x_{2}-\theta_{1}^{h}\right]^{T} \\
C_{i j k l}^{B} \mapsto \mathbf{C}^{B}=\frac{E t^{3}}{12\left(1-\nu^{2}\right)}\left[\begin{array}{ccc}
1 & \nu & 0 \\
\nu & 1 & 0 \\
0 & 0 & \frac{1-\nu}{2}
\end{array}\right] ; C_{i j}^{S} \mapsto \mathbf{C}^{S}=\frac{c E t}{2(1+\nu)}\left[\begin{array}{cc}
1 & 0 \\
0 & 1
\end{array}\right]
\end{gathered}
$$

In (2.8) $t$ is plate thickness, $E$ is Young's modulus and $\nu$ is Poisson's ratio. The shear correction factor $c$ is usually set to $5 / 6$ (see [5] or [7]). Superscript $h$ in (2.5) is the mesh parameter, which is usually used to denote the quantities in the discrete approximation.

In what follows we will restrict ourselves to quadrilateral plate elements. The discretized domain $\Omega^{h}$ is represented by a finite element mesh of plate elements, $\Omega^{h}=\bigcup_{e=1}^{n_{e l}} \Omega^{e}$, where $n_{e l}$ is the number of elements in the mesh. The geometry of an element is defined by the bilinear mapping $\boldsymbol{\xi} \in \square \mapsto$ $\mathbf{x}^{h} \in \Omega^{e}$

$$
\left.\mathbf{x}^{h}(\boldsymbol{\xi})\right|_{\Omega^{e}}=\sum_{I=1}^{4} N_{I}(\boldsymbol{\xi}) \mathbf{x}_{I} ; \quad \mathbf{x}_{I}=\left[x_{1 I} ; x_{2 I}\right]^{T} ; \quad \boldsymbol{\xi}=[\xi, \eta]
$$

where $\square \in[-1,+1] \times[-1,+1], \mathbf{x}_{I}$ are coordinates of a finite element node $I$, and

$$
N_{I}(\xi, \eta)=\frac{1}{4}\left(1+\xi_{I} \xi\right)\left(1+\eta_{I} \eta\right) \quad \begin{array}{c|c|c|c|c}
\mathrm{I} & 1 & 2 & 3 & 4 \\
\hline \xi_{I} & -1 & +1 & +1 & -1 \\
\hline \eta_{I} & -1 & -1 & +1 & +1
\end{array}
$$

\subsection{Reissner-Mindlin quadrilateral (RMQ) plate ele- ment}

Interpolation of the rotation field is based on quadratic polynomials (see Figure 1 and [12] for details)

$$
\left(\begin{array}{c}
\theta_{1}^{h} \\
\theta_{2}^{h}
\end{array}\right)=\left.\boldsymbol{\theta}^{h}(\boldsymbol{\xi})\right|_{\Omega^{e}}=\sum_{I=1}^{4} N_{I}(\boldsymbol{\xi}) \boldsymbol{\theta}_{I}+\sum_{L=5}^{8} N_{L}(\boldsymbol{\xi}) \boldsymbol{n}_{J K} \Delta \theta_{J K}
$$


where

$$
\begin{array}{ll}
N_{L}(\boldsymbol{\xi})=\frac{1}{2}(1-\xi)^{2}\left(1+\eta_{J} \eta\right) ; & \mathrm{L}=5,7 \\
N_{L}(\boldsymbol{\xi})=\frac{1}{2}(1-\eta)^{2}\left(1+\xi_{J} \xi\right) ; & \mathrm{L}=6,8
\end{array}
$$

\begin{tabular}{c|c|c|c|c}
$\mathrm{L}$ & 5 & 6 & 7 & 8 \\
\hline $\mathrm{J}$ & 1 & 2 & 3 & 4 \\
\hline $\mathrm{K}$ & 2 & 3 & 4 & 1
\end{tabular}

and

$$
\left.\boldsymbol{n}_{J K}=\left[\cos \alpha_{J K} ; \sin \alpha_{J K}\right]^{T} ; \quad l_{J K}=\left(\left(x_{1 K}-x_{1 J}\right)^{2}\right)+\left(x_{2 K}-x_{2 J}\right)^{2}\right)^{1 / 2}
$$

Location of rotations $\theta_{I}$ and $\Delta \theta_{J K}$ is illustrated in Figure 1. Displacement field is interpolated by cubic polynomials

$$
\begin{gathered}
\left.w^{h}(\boldsymbol{\xi})\right|_{\Omega^{e}}=\sum_{I=1}^{4} N_{I}(\boldsymbol{\xi}) w_{I}+\sum_{L=5}^{8} N_{L}(\boldsymbol{\xi}) \frac{l_{J K}}{8} \mathbf{n}_{J K}^{T}\left(\boldsymbol{\theta}_{J}-\boldsymbol{\theta}_{K}\right)+\sum_{L=5}^{8} M_{L}(\boldsymbol{\xi}) \frac{l_{J K}}{6} \Delta \theta_{J K} \\
M_{L}(\boldsymbol{\xi})=\frac{1}{2} \eta_{J}(1-\xi)^{2} \xi\left(1+\eta_{J} \eta\right) ; \quad \mathrm{L}=5,7 \\
M_{L}(\boldsymbol{\xi})=\frac{1}{2} \xi_{J}(1-\eta)^{2} \eta\left(1+\xi_{J} \xi\right) ; \quad \mathrm{L}=6,8
\end{gathered}
$$

Interpolation of bending strains follows from (2.6) and (2.11)

$$
\begin{gathered}
\left.\boldsymbol{\kappa}^{h}(\boldsymbol{\xi})\right|_{\Omega^{e}}=\sum_{I=1}^{4} \mathbf{B}_{I}(\boldsymbol{\xi}) \boldsymbol{\theta}_{I}+\sum_{L=5}^{8} \tilde{\mathbf{B}}_{L} \boldsymbol{n}_{J K} \Delta \theta_{J K} \\
\mathbf{B}_{I}=\left[\begin{array}{cc}
0 & -N_{I, x_{1}} \\
+N_{I, x_{2}} & 0 \\
+N_{I, x_{1}} & -N_{I, x_{2}}
\end{array}\right] ; \quad \tilde{\mathbf{B}}_{L}=\left[\begin{array}{cc}
0 & -N_{L, x_{1}} \\
+N_{L, x_{2}} & 0 \\
+N_{L, x_{1}} & -N_{L, x_{2}}
\end{array}\right]
\end{gathered}
$$

where notation $(\cdot)_{x_{i}}=\frac{\partial(\cdot)}{\partial \xi_{j}} \frac{\partial \xi_{j}}{\partial x_{i}} ; \xi_{1}=\xi ; \xi_{2}=\eta$ is used. We further choose a bilinear distribution for the assumed shear strain in the form

$$
\left(\begin{array}{c}
\gamma_{1}^{h} \\
\gamma_{2}^{h}
\end{array}\right)=\left.\gamma^{h}(\boldsymbol{\xi})\right|_{\Omega^{e}}=\sum_{I=1}^{4} N_{I}(\boldsymbol{\xi}) \gamma_{I}
$$

where nodal shear strains $\gamma_{I}$ are consistent with the constant shear strain distribution along each edge. For a node $I$ we have

$$
\gamma_{I}=\frac{1}{\mathbf{t}_{I J}^{T} \mathbf{n}_{I K}}\left[\frac{1}{l_{I K}} \mathbf{n}_{I J} w_{K}+\frac{1}{l_{I J}} \mathbf{n}_{I K} w_{J}-\left(\frac{1}{l_{I K}} \mathbf{n}_{I J}+\frac{1}{l_{I J}} \mathbf{n}_{I K}\right) w_{I}\right.
$$




$$
\begin{aligned}
& +\frac{1}{2} \mathbf{n}_{I J} \mathbf{n}_{I K}^{T} \boldsymbol{\theta}_{K}-\frac{1}{2} \mathbf{n}_{I K} \mathbf{n}_{I J}^{T} \boldsymbol{\theta}_{J}+\frac{1}{2}\left(\mathbf{n}_{I J} \mathbf{n}_{I K}^{T}-\mathbf{n}_{I K} \mathbf{n}_{I J}^{T}\right) \boldsymbol{\theta}_{I} \\
& \left.+\frac{2}{3} \mathbf{n}_{I J} \Delta \theta_{I K}-\frac{2}{3} \mathbf{n}_{I K} \Delta \theta_{I J}\right] ; \quad \begin{array}{r|r|r|r|r|r}
\hline \mathrm{J} & 4 & 1 & 2 & 3 \\
\hline \mathrm{K} & 2 & 3 & 4 & 1
\end{array}
\end{aligned}
$$

Notation for strains can be further simplified by using

$$
\left.\boldsymbol{\kappa}^{h}(\boldsymbol{\xi})\right|_{\Omega^{e}}=\sum_{I=1}^{4} \widehat{\mathbf{B}}_{I} \mathbf{u}_{I},\left.\quad \boldsymbol{\gamma}^{h}(\boldsymbol{\xi})\right|_{\Omega^{e}}=\sum_{I=1}^{4} \mathbf{G}_{I} \mathbf{u}_{I}, \quad \mathbf{u}_{I}=\left(\begin{array}{c}
w_{I} \\
\boldsymbol{\theta}_{I} \\
\Delta \theta_{J K}
\end{array}\right)
$$

where $\hat{\boldsymbol{B}}_{I}$ follows from (2.14) and $\boldsymbol{G}_{I}$ from (2.15) and (2.16).
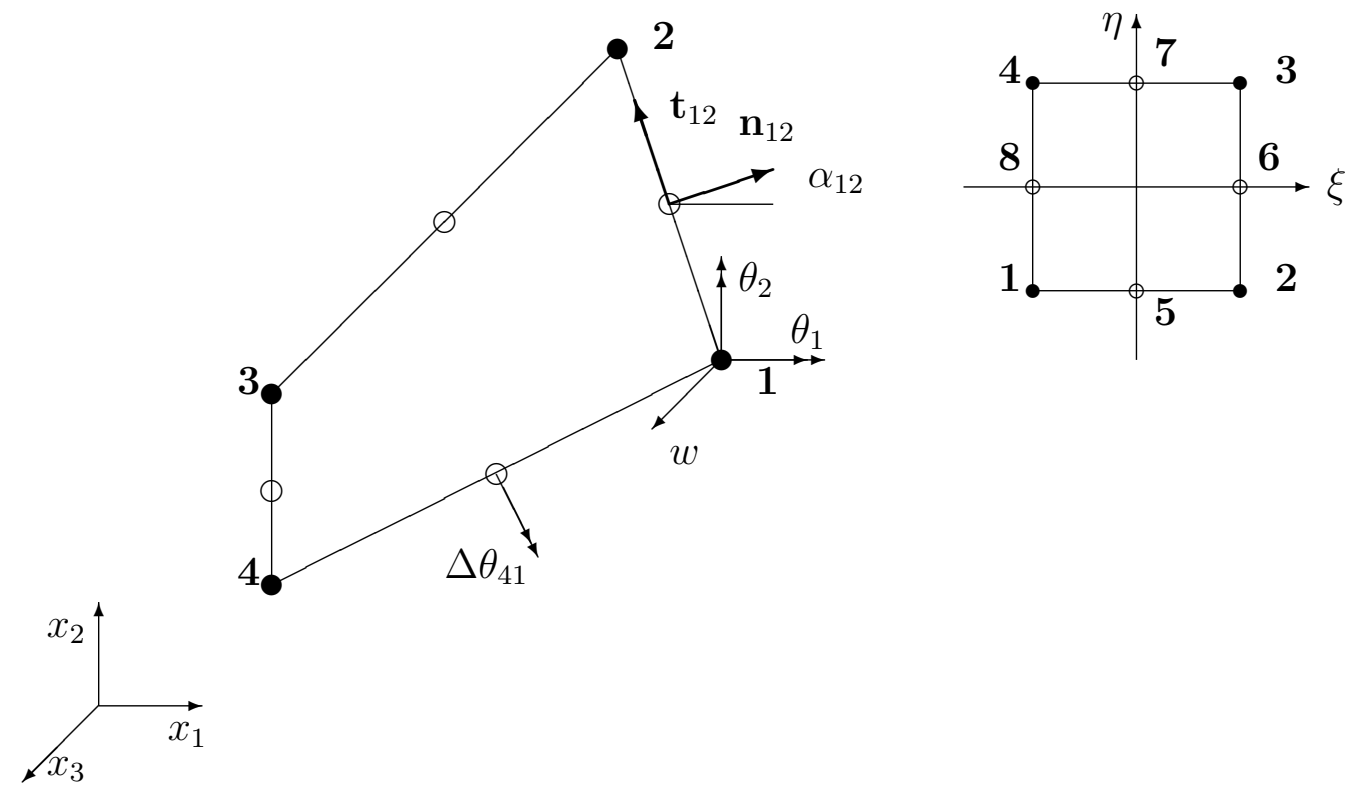

Figure 1: Plate element with cubic displacement interpolation 
The element stiffness matrix can be computed on the node to node basis as

$$
\mathbf{K}_{I J}^{e}=\int_{\Omega^{e}} \widehat{\mathbf{B}}_{I}^{T}(\boldsymbol{\xi}) \mathbf{C}^{B} \widehat{\mathbf{B}}_{J}(\boldsymbol{\xi}) d \Omega+\int_{\Omega^{e}} \mathbf{G}_{I}^{T}(\boldsymbol{\xi}) \mathbf{C}^{S} \mathbf{G}_{J}(\boldsymbol{\xi}) d \Omega
$$

Both bending and shear strains have been taken into account. The presented plate element has 8 nodes and 16 dofs, 3 per each vertex node and 1 hierarchic rotation $\Delta \theta_{I J}$ at each mid-side node.

Remark: Reissner-Mindlin plate with only 3 dofs per node and incompatible internal dofs can be developed by using the method of incompatible modes and by eliminating the relative rotations at the element level (see [13]).

\subsection{Discrete Kirchhoff quadrilateral (DKQ) plate ele- ment}

On the basis of Reissner-Mindlin quadrilateral of the preceding section one can derive the DKQ element. If we impose a constraint that the shear strain

vanishes along all element edges (see [12] for details), we can express the hierarchical rotations as

$$
\Delta \theta_{J K}=\frac{3}{2 l_{J K}}\left(w_{K}-w_{J}\right)-\frac{3}{4} \mathbf{n}_{J K} \cdot\left(\boldsymbol{\theta}_{J}+\boldsymbol{\theta}_{K}\right)
$$

The discrete approximation (2.11) can then be written as

$$
\begin{aligned}
\left.\boldsymbol{\theta}^{h}(\boldsymbol{\xi})\right|_{\Omega^{e}}= & \sum_{I=1}^{4} N_{I}(\boldsymbol{\xi}) \boldsymbol{\theta}_{I} \\
& +\sum_{L=5}^{8} N_{L}(\boldsymbol{\xi})\left[\frac{3}{2 l_{J K}} \mathbf{n}_{J K}\left(w_{K}-w_{J}\right)-\frac{3}{4} \mathbf{n}_{J K} \mathbf{n}_{J K}^{T}\left(\boldsymbol{\theta}_{J}+\boldsymbol{\theta}_{K}\right)\right]
\end{aligned}
$$

and the explicit form of the discrete approximation of the displacement field (2.2) reads

$$
\begin{aligned}
\left.w^{h}(\boldsymbol{\xi})\right|_{\Omega^{e}}= & \sum_{I=1}^{4} N_{I}(\boldsymbol{\xi}) w_{I}+\sum_{L=5}^{8} N_{L}(\boldsymbol{\xi}) \frac{l_{J K}}{8} \mathbf{n}_{J K}^{T}\left(\boldsymbol{\theta}_{J}-\boldsymbol{\theta}_{K}\right) \\
& +\sum_{L=5}^{8} M_{L}(\boldsymbol{\xi})\left[\frac{1}{4}\left(w_{K}-w_{J}\right)-\frac{l_{J K}}{8} \mathbf{n}_{J K}^{T}\left(\theta_{J}+\theta_{K}\right)\right]
\end{aligned}
$$


The displacement and rotation finite element interpolations (2.19) and (2.20) will make nodal shear strains (2.16) equal to zero. Due to (2.15), the shear strain discrete approximation is zero throughout the whole element domain. The element stiffness matrix and load vector for such a plate element can be computed on the node to node basis as

$$
\boldsymbol{K}_{I J}^{e}=\int_{\Omega^{e}} \overline{\mathbf{B}}_{I}^{T}(\boldsymbol{\xi}) \mathbf{C}^{B} \overline{\mathbf{B}}_{J}(\boldsymbol{\xi}) d \Omega, \quad \mathbf{f}_{I}=\int_{\Omega^{e}} \widehat{\mathbf{N}}_{I}^{T}(\boldsymbol{\xi}) f d \Omega
$$

where

$$
\left.w^{h}\right|_{\Omega^{e}}=\sum_{I=1}^{4} \widehat{\mathbf{N}}_{I}^{T} \mathbf{u}_{I},\left.\quad \boldsymbol{\kappa}^{h}\right|_{\Omega^{e}}=\sum_{I=1}^{4} \overline{\mathbf{B}}_{I} \mathbf{u}_{I}, \quad \mathbf{u}_{I}=\left(\begin{array}{c}
w_{I} \\
\boldsymbol{\theta}_{I}
\end{array}\right)
$$

$\overline{\mathbf{B}}_{I}$ follows from (2.14) and (2.18) and $\widehat{\mathbf{N}}_{I}$ follows from (2.20). Note, that this 4 node quadrilateral element has only 3 dofs per node.

\subsection{Discretization error and mesh adaptivity}

In order to control the mesh density through the mesh adaptivity one has to estimate the discretization error. Since the main interest of the plate finite element analysis are stress resultants, the a posteriori discretization error can be estimated by the energy norm that uses the difference between the "true" and the finite element (FE) stress resultants. The "true" ones are obviously not known, but one can produce their estimate by improving the computed values of FE stress resultants. This can be achieved by smoothing the FE stress resultants across the whole mesh, looking for the best possible fit of smoothed solution in the least square sense to the FE solution. The computation of this kind can be carried out independently for each stress resultant component.

Let us illustrate the procedure for bending moment component $m_{i j}^{h}$. The FE results are available at each Gauss point of each plate element $\left.m_{i j}^{h}\left(\boldsymbol{\xi}_{G}\right)\right|_{\Omega^{e}}=$ $\left.C_{i j k l}^{B} \kappa_{k l}^{h}\left(\boldsymbol{\xi}_{G}\right)\right|_{\Omega^{e}}$. The corresponding smooth field can be obtained over each element by using the standard bilinear shape functions $N_{I}(\boldsymbol{\xi})$ and unknown nodal values $a_{I}$,

$$
\left.m_{i j}^{*}(\boldsymbol{\xi})\right|_{\Omega^{e}}=\sum_{I=1}^{4} N_{I}(\boldsymbol{\xi}) a_{I}
$$


The least squares fit results in

$$
\frac{1}{2} \sum_{e=1}^{n_{e l}} \int_{\Omega^{e}}\left[m_{i j}^{*}(\boldsymbol{\xi})-m_{i j}^{h}(\boldsymbol{\xi})\right]^{2} d \Omega \mapsto \min
$$

The last two equations allow us to write

$$
\mathcal{A}_{e=1}^{n_{e l}[}\left[M_{I J}^{e}\right] a_{J}=\mathcal{A}_{e=1}^{n_{e l}} b_{I}^{e}
$$

with

$$
\begin{gathered}
M_{I J}^{e}=\int_{\Omega^{e}} N_{I}(\boldsymbol{\xi}) N_{J}(\boldsymbol{\xi}) d \Omega=\sum_{G=1}^{n i n t} N_{I}\left(\boldsymbol{\xi}_{G}\right) N_{J}\left(\boldsymbol{\xi}_{G}\right) j\left(\boldsymbol{\xi}_{G}\right) w_{G} \\
b_{I}^{e}=\int_{\Omega^{e}} N_{I}(\boldsymbol{\xi}) m_{i j}^{h} d \Omega=\sum_{G=1}^{n i n t} N_{I}\left(\boldsymbol{\xi}_{G}\right) m_{i j}^{h}\left(\boldsymbol{\xi}_{G}\right) j\left(\boldsymbol{\xi}_{G}\right) w_{G}
\end{gathered}
$$

where $\mathcal{A}_{e=1}^{n_{e l}}$ denotes finite element assembly procedure, nint is number of element integration points, $w_{G}$ are weights of integration points, and $j$ is Jacobian of transformation $\square \mapsto \Omega^{e}$.

Different proposals are made in literature in order to reduce the computational cost of this global problem for computing the smooth approximation. For example, one can use it only at the level on a single element (e.g. [11]), or at the level of a patch of elements surrounding a particular node (e.g. [30]), or yet at the global level but using only diagonal form of matrix $\mathbf{M}$ to make the computation more efficient (e.g. [29]).

The discretization error can be estimated by the energy norm. For the DKQ it can be based on the difference between the improved moments $\mathbf{m}^{*}$ and the moments $\mathbf{m}=C^{B} \kappa^{h}$ available from the proceeding FE computation

$$
\|e\|^{2}=\int_{\Omega^{h}}\left(\mathbf{m}^{*}-\mathbf{m}\right)^{T} \mathbf{C}^{B^{-1}}\left(\mathbf{m}^{*}-\mathbf{m}\right) d \Omega
$$

For the RMQ other strategies can be applied that take into account also the influence of boundary layers, see e.g. [18]. Integral over the whole domain (2.22) can be decomposed into element integrals and the total error estimator can be expressed as the sum of element error estimators

$$
\|e\|^{2}=\sum_{e}\left\|e_{e}\right\|^{2}
$$


This information can be used to obtain each element contribution to the total discretization error, as well as to estimate the need to refine the mesh.

In order to construct optimal mesh with uniform distribution of discretization error, one needs to relate element size with the discretization error.

Typical element size $h_{e}$ can be defined as

$$
h_{e}=\sqrt{A_{e} / \pi}
$$

where $A_{e}$ is element area. From the a priori analysis of discretization error we get (see [29]) the following relation

$$
\left\|e_{e}\right\|=C h_{e}^{p}
$$

where $p$ stands for the polynomial order of used interpolation and $C$ is size independent constant.

The aim is to construct a mesh with $N_{e}$ elements and a uniform distribution of discretization error with its total value $\|e\| \approx T O L$. Following (2.23) the element discretization error should then be

$$
\left\|e_{e}\right\| \approx \frac{T O L}{\sqrt{N_{e}}}
$$

Desired element size can thus be determined from (2.24) as

$$
\tilde{h}_{e}=\left(\frac{T O L}{C \sqrt{N_{e}}}\right)^{(1 / p)}
$$

Since the estimate of discretization error $\left\|e_{e}\right\|$ for a element of size $h_{e}$ is known, the value of constant can be deduced from it

$$
C=\left\|e_{e}\right\| / h_{e}^{p}
$$

Estimated new size of the element is then

$$
\tilde{h}_{e}=h_{e}\left(\frac{T O L}{\left\|e_{e}\right\| \sqrt{N_{e}}}\right)^{(1 / p)}
$$

Based on obtained estimate of element size distribution the new mesh is automatically generated. Mesh refinement is repeated until the desired accuracy is met. 


\section{Model adaptivity for plates}

To locate regions of the domain where the chosen mathematical model (usually the simplest one in a set of available hierarchic models) no longer performs well, one has to provide an estimate of model performance. Ideally, such an estimate for the chosen model should follow from the comparison with the best possible mathematical model (which is often the $2 \mathrm{D} / 3 \mathrm{D}$ solid model). However, the best model estimate is in general not feasible, since it remains prohibitively expensive or simply inaccessible. Thus, for the practical model error estimation, it is sufficient to compare the chosen model with the one which is known to perform better; the latter will be called the enhanced model.

In principle, two global computations would be required to compare two different mathematical models: the chosen against the enhanced one. However, the computations with the enhanced model is usually simplified in trying to estimate the true stress state. This is made possible by extracting a portion of the domain, reducing the computation to a single finite element based on the enhanced model, applying the loading on its edges according to the true stress state estimate, computing the local enhanced solution and comparing it to the original solution obtained by the chosen model. This is of course possible only in principle, since the true stress state is unknown. However, its approximation can be obtained by improving the FE solution obtained with the chosen model to be best-possible approximation of the true stress state. This is done as follows: the FE solution for any stress component (which is discontinuous between the elements) is improved to be continuous in order to approximate the true stress state, which is continuous (unless there is plate thickness, loading or material discontinuity). In accordance with the best-possible approximation of the true stress state, the edge loading (so-called boundary traction) for each finite element of the mesh is computed. That loading is further used in computation of local (elementwise) Neumann problems based on the enhanced model. Comparison of two mathematical models can be thus achieved by one global and a set of local computations.

In the case of plate, the above described procedure implies the need for a hierarchic family of plate models, which should be established by means of plate model comparison with the 3D solid model (e.g. see [4]). To construct the boundary stress resultants (which are plate counterparts of solid boundary traction) we follow the procedure outlined e.g in [15], [16], [25], [21] for 
3D elasticity. The local problem that needs to be solved (to get a model error indicator/estimator) deals with single plate element, which is floating structure, loaded on the surface and along its edges in such a way that the loads are self-equilibrated. We can thus speak about the model adaptivity procedure for plates that is based on equilibrated boundary stress resultants.

\subsection{Construction of equilibrated boundary tractions for $2 \mathrm{D} / 3 \mathrm{D}$ solid}

The idea of equilibrated boundary traction (edge loading) comes from the observation that the finite element equilibrium equation for a single element can be written as

$$
\mathbf{f}^{\text {int }, e}-\mathbf{f}^{e x t, e}=\mathbf{r}^{e}
$$

where $\mathbf{f}^{e x t, e}, \mathbf{r}^{e}$ and $\mathbf{f}^{\text {int,e }}$ are nodal values of external, residual and internal forces, respectively. The later are proportional to the stiffness matrix $\mathbf{K}^{e}$ and the nodal displacements $\mathbf{d}^{e}$, so that (3.1) becomes:

$$
\mathbf{K}^{e} \mathbf{d}^{e}=\mathbf{f}^{e x t, e}+\mathbf{r}^{e}
$$

Residual forces can be seen as the action of the surrounding elements.

The goal is to find such boundary traction forces that exactly replace the residual forces and are continuous across element boundaries. We thus seek for boundary traction $\mathbf{t}_{\Gamma}^{e}$ acting on an edge $\Gamma(\Gamma \subset \partial \Omega e$, where $\partial \Omega e$ denotes element boundary), which will replace (in an energy manner) the action of residual nodal forces and reflect the continuity of the stress field. We can write

$$
\begin{gathered}
\sum_{I} \mathbf{r}_{I}^{e} \cdot \hat{\mathbf{v}}_{I}^{h}=\sum_{\Gamma} \int_{\Gamma} \mathbf{t}_{\Gamma}^{e} \cdot \hat{\mathbf{v}}_{\Gamma}^{h} d s \\
\mathbf{t}_{\Gamma}^{e}+\mathbf{t}_{\Gamma}^{e^{\prime}}=0
\end{gathered}
$$

where $\widehat{\mathbf{v}}^{h}=\sum N_{I} \hat{\mathbf{v}}_{I}^{h}$ are element virtual displacements, $\hat{\mathbf{v}}_{\Gamma}^{h}=\left.\widehat{\mathbf{v}}^{h}\right|_{\Gamma}$ are edge virtual displacements, and $\hat{\mathbf{v}}_{I}^{h}$ are nodal virtual displacements. Elements $e$ and $e^{\prime}$ share the same edge $\Gamma$. We note that the condition in (3.3) follows from $\mathbf{t}_{\Gamma}^{e}=\boldsymbol{\sigma} \mathbf{n}^{e}$ and $\mathbf{n}^{e^{\prime}}=-\mathbf{n}^{e}$, where $\mathbf{n}$ is normal to the edge $\Gamma$ and $\boldsymbol{\sigma}$ is continuous stress tensor.

We further express the nodal value of the element residual force $\mathbf{r}_{I}^{e}$ in (3.2) as the sum

$$
\mathbf{r}_{I}^{e}=\mathbf{r}_{I, \Gamma_{1}}^{e}+\mathbf{r}_{I, \Gamma_{2}}^{e}
$$


where $\Gamma_{1}$ and $\Gamma_{2}$ are two edges of element $e$ that have in common node $I$. We can see $\mathbf{r}_{I, \Gamma}^{e}$ as residual forces at node $I$ of element $e$ due to the boundary traction applied on edge $\Gamma$, i.e.

$$
\mathbf{r}_{I, \Gamma}^{e}=\frac{\partial W_{\Gamma}^{e}\left(\mathbf{t}_{\Gamma}^{e}\right)}{\partial \hat{\mathbf{v}}_{I}^{h}} ; \quad W_{\Gamma}^{e}\left(\mathbf{t}_{\Gamma}^{e}\right)=\int_{\Gamma} \mathbf{t}_{\Gamma}^{e} \cdot \hat{\mathbf{v}}_{\Gamma}^{h} d s
$$

By collecting equations (3.4) and (3.3) for all elements in the mesh $(e=$ $\left.1, \ldots, n_{e l}\right)$, all element edges $\left(\Gamma=1, \ldots n_{\Gamma}\right)$ and all element nodes $(I=$ $\left.1, \ldots n_{e n}\right)$, we get a global system of equations for the unknowns $\mathbf{r}_{I, \Gamma}^{e}$ (the number of unknowns in such a system is reduced if there are regions of the discretized domain where boundary traction forces are prescribed).

In order to get further insight into the procedure for computation of $\mathbf{r}_{I, \Gamma}^{e}$, we inspect a patch of four $2 \mathrm{D}$ elements surrounding node $I$ of the FE mesh (see Figure 2). Edges and elements are numbered in counter-clockwise manner. Application of equation (3.4) for this case gives

$$
\begin{aligned}
\mathbf{r}_{I}^{e_{1}} & =\mathbf{r}_{I, \Gamma_{4}}^{e_{1}}+\mathbf{r}_{I, \Gamma_{1}}^{e_{1}} \\
\mathbf{r}_{I}^{e_{2}} & =\mathbf{r}_{I, \Gamma_{1}}^{e_{2}}+\mathbf{r}_{I, \Gamma_{2}}^{e_{2}} \\
\mathbf{r}_{I}^{e_{3}} & =\mathbf{r}_{I, \Gamma_{2}}^{e_{3}}+\mathbf{r}_{I, \Gamma_{3}}^{e_{3}} \\
\mathbf{r}_{I}^{e_{4}} & =\mathbf{r}_{I, \Gamma_{3}}^{e_{4}}+\mathbf{r}_{I, \Gamma_{4}}^{e_{4}}
\end{aligned}
$$

The demand for continuity further leads to

$$
\begin{aligned}
& \mathbf{r}_{I, \Gamma_{1}}^{e_{1}}+\mathbf{r}_{I, \Gamma_{1}}^{e_{2}}=0 \\
& \mathbf{r}_{I, \Gamma_{2}}^{e_{2}}+\mathbf{r}_{I, \Gamma_{2}}^{e_{3}}=0 \\
& \mathbf{r}_{I, \Gamma_{3}}^{e_{3}}+\mathbf{r}_{I, \Gamma_{3}}^{e_{4}}=0 \\
& \mathbf{r}_{I, \Gamma_{4}}^{e_{4}}+\mathbf{r}_{I, \Gamma_{4}}^{e_{1}}=0
\end{aligned}
$$

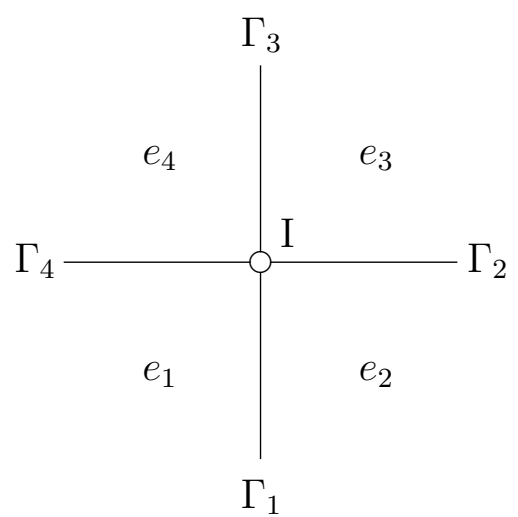

Figure 2: A patch of four elements surrounding node $I$

Combination of the last two sets of equations leads to the following system

$$
\left[\begin{array}{c}
\mathbf{r}_{I}^{e_{1}} \\
\mathbf{r}_{I}^{e_{2}} \\
\mathbf{r}_{I}^{e_{3}} \\
\mathbf{r}_{I}^{e_{4}}
\end{array}\right]=\left[\begin{array}{cccc}
+1 & 0 & 0 & -1 \\
-1 & +1 & 0 & 0 \\
0 & -1 & +1 & 0 \\
0 & 0 & -1 & +1
\end{array}\right]\left[\begin{array}{c}
\mathbf{r}_{I, \Gamma_{1}}^{e_{1}} \\
\mathbf{r}_{I, \Gamma_{2}}^{e_{2}} \\
\mathbf{r}_{I, \Gamma_{3}}^{e_{3}} \\
\mathbf{r}_{I, \Gamma_{4}}^{e_{4}}
\end{array}\right]
$$

for the unknowns $\mathbf{r}_{I, \Gamma_{k}}^{e_{k}}$. Since all the unknowns refer only to node $I$ of the FE mesh, the system is independent from other nodes. One thus obtains 
a local (patch-wise) problem; a global system is actually a composition of independent patch-wise systems. If local system (3.6) is solved for each node of the FE mesh, we obtain $\mathbf{r}_{I, \Gamma}^{e_{k}}$, for all elements of the mesh $\left(e_{k}=e=\right.$ $\left.1, \ldots, n_{e l}\right)$, all element edges $\left(\Gamma=1, \ldots n_{\Gamma}\right)$ and all element nodes $(I=$ $\left.1, \ldots n_{e n}\right)$.

If $\mathbf{r}_{I, \Gamma}^{e}$ are known, the boundary traction can be defined for each element as follows. We introduce parametrization of $k$-th component of the boundary traction $\mathbf{t}_{\Gamma}^{e}$ (let $\Gamma$ be element edge between element nodes $I$ and $J$ ) as

$$
\left(t_{\Gamma}^{e}\right)_{k}=p_{k}^{I} \psi_{k}^{I}+p_{k}^{J} \psi_{k}^{J}
$$

where $\psi_{k}^{I}$ are shape functions (yet to be chosen) and $p_{k}^{I}$ are nodal values of $\left(t_{\Gamma}^{e}\right)_{k}$. The variation of virtual displacements along the same edge $\Gamma$ can be written as

$$
\left(\widehat{v}_{\Gamma}^{h}\right)_{k}=N_{k, m}^{I} \hat{v}_{m}^{I}+N_{k, m}^{J} \hat{v}_{m}^{J}
$$

where $\hat{v}_{m}^{I}$ is related to the $m$-th degree of freedom at node $I$, and $N_{k, m}^{I}$ are the corresponding shape functions. In other words, the shape function $N_{k, m}^{I}$ interpolates nodal value $\hat{v}_{m}^{I}$ for the $k$-th component of virtual displacements. We can now insert the above parameterizations into (3.5) and express residuals at nodes $I$ and $J$ with respect to the boundary traction on edge $\Gamma$ (that spans between $I$ and $J$ ). The result can be written in a compact form as:

$$
\left[\begin{array}{c}
\mathbf{r}_{I, \Gamma}^{e} \\
\mathbf{r}_{J, \Gamma}^{e}
\end{array}\right]=\left[\begin{array}{ll}
\mathbf{M}_{\Gamma}^{I I} & \mathbf{M}_{\Gamma}^{I J} \\
\mathbf{M}_{\Gamma}^{J I} & \mathbf{M}_{\Gamma}^{J J}
\end{array}\right]\left[\begin{array}{c}
\mathbf{p}_{\Gamma}^{I} \\
\mathbf{p}_{\Gamma}^{J}
\end{array}\right] ; \quad \mathbf{M}_{\Gamma, n m}^{I J}=\int_{\Gamma} N_{m, n}^{I} \psi_{m}^{J} d s
$$

where $\mathbf{p}_{\Gamma}^{I}=\left[p_{k}^{I}, k=1, \ldots n_{\operatorname{dim}}\right]^{T}$, and $n_{\text {dim }}$ is vector dimension. By solving (3.9) for every element edge $\Gamma$, we obtain parameters $\mathbf{p}_{\Gamma_{I J}}^{I}$, which define element boundary traction $\mathbf{t}_{\Gamma}^{e}$ through (3.7).

It is suggested in the literature (e.g. [1], [21]) that shape functions $\psi_{k}^{J}$ and $\psi_{k}^{I}$ should be such that $\mathbf{M}_{\Gamma}^{I J}$ and $\mathbf{M}_{\Gamma}^{J I}$ vanish and the matrix $\mathbf{M}$ becomes block diagonal. In such a case (3.9) simplifies to

$$
\mathbf{r}_{I, \Gamma}^{e}=\mathbf{M}_{\Gamma}^{I I} \mathbf{p}_{\Gamma}^{I}
$$

This makes the bookkeeping for the computations much easier. However, our experience is that the form of boundary traction obtained in this way is very specific and it does not represent well the stress field. We also note that the specific choice of parametrization (3.10) is not necessary, since local patch-wise computation can be performed, see (3.6). 


\subsection{Regularization of local system in (3.6)}

The solution of local system of equations (3.6) is not unique, unless we impose an adequate additional regularization. The latter can be derived in accordance with the estimate of the true stress state with the continuous boundary traction across the element interfaces. However, the true stress state is not known, but only its (discontinuous) element-wise approximations $\boldsymbol{\sigma}_{F E}^{e}$. Continuous stresses at the edge $\Gamma$ denoted as $\left.\boldsymbol{\sigma}_{0}\right|_{\Gamma}$ follow from the smooth stress recovery which may be coupled with previous mesh adaptivity procedure. Boundary traction forces resulting from a such stresses $\left.\boldsymbol{\sigma}_{0}\right|_{\Gamma}$ are obtained by

the use of the Cauchy principle $\boldsymbol{t}_{\Gamma}^{0, e}=\left.\boldsymbol{\sigma}_{0}\right|_{\Gamma} \boldsymbol{n}^{e}$. It is now possible to calculate the effect of this edge loading on neighboring nodes by using (3.5)

$$
\mathbf{r}_{I, \Gamma}^{e, 0}=\frac{\partial W_{\Gamma}^{e}\left(\boldsymbol{t}_{\Gamma}^{0, e}\right)}{\partial \hat{\boldsymbol{v}}_{I}^{h}}
$$

We want $\mathbf{r}_{I, \Gamma}^{e}$ to be as close as possible to this result in the least square sense. We construct the following constrained patch-wise minimization problem:

$$
\begin{aligned}
\mathcal{L}\left\{\mathbf{r}_{I, \Gamma}^{e} ; \lambda^{\Gamma} ; \sigma^{e}\right\} & =\frac{1}{2} \sum_{e \in \mathcal{P}_{I}} \sum_{\Gamma}\left(\mathbf{r}_{I, \Gamma}^{e, 0}-\mathbf{r}_{I, \Gamma}^{e}\right)^{2} \\
& +\sum_{e \in \mathcal{P}_{I}} \sigma_{I}^{e}\left(\mathbf{r}_{I}^{e}-\sum_{\Gamma} \mathbf{r}_{I, \Gamma}^{e}\right) \\
& +\sum_{\Gamma} \lambda_{I}^{\Gamma}\left(\mathbf{r}_{I, \Gamma}^{e}+\mathbf{r}_{I, \Gamma}^{e^{\prime}}\right)
\end{aligned}
$$

where the constraints (3.3) and (3.4) are also introduced by means of the corresponding Lagrange multipliers $\sigma_{I}^{e}$ and $\lambda_{I}^{\Gamma}$, and $\mathcal{P}_{I}$ is patch of elements around node $I$ of the FE mesh.

The Kuhn-Tucker optimality conditions for stationary point are then given by differentiating the Lagrangian with respect to unknowns $\mathbf{r}_{I, \Gamma}^{e}$ :

$$
\mathbf{r}_{I, \Gamma}^{e}-\mathbf{r}_{I, \Gamma}^{e, 0}-\sigma_{I}^{e}+\lambda_{I}^{\Gamma}=0
$$

The same equation also holds for the residual force on the neighboring element $\mathbf{r}_{I, \Gamma}^{e^{\prime}}$ :

$$
\mathbf{r}_{I, \Gamma}^{e^{\prime}}-\mathbf{r}_{I, \Gamma}^{e^{\prime}, 0}-\sigma_{I}^{e^{\prime}}+\lambda_{I}^{\Gamma}=0
$$


If we sum up the last two equations and take into account the continuity condition $\mathbf{r}_{I, \Gamma}^{e}+\mathbf{r}_{I, \Gamma}^{e^{\prime}}=0$ we can express the Lagrange multiplier $\lambda_{I}^{\Gamma}$ as

$$
\lambda_{I}^{\Gamma}=\frac{1}{2}\left(\sigma_{I}^{e}+\sigma_{I}^{e^{\prime}}+\mathbf{r}_{I, \Gamma}^{e, 0}+\mathbf{r}_{I, \Gamma}^{e^{\prime}, 0}\right)
$$

The unknowns $\mathbf{r}_{I, \Gamma}^{e}$ can then be expressed in terms of the multiplier $\sigma_{I}^{e}$

$$
\mathbf{r}_{I, \Gamma}^{e}=\frac{1}{2}\left(\sigma_{I}^{e}-\sigma_{I}^{e^{\prime}}+\mathbf{r}_{I, \Gamma}^{e, 0}-\mathbf{r}_{I, \Gamma}^{e^{\prime}, 0}\right)
$$

The condition $\mathbf{r}_{I}^{e}=\mathbf{r}_{I, \Gamma_{1}}^{e}+\mathbf{r}_{I, \Gamma_{2}}^{e}$ can now be rewritten as

$$
\mathbf{r}_{I}^{e}=\frac{1}{2}\left(\mathbf{r}_{I, \Gamma_{1}}^{e, 0}-\mathbf{r}_{I, \Gamma_{1}}^{e_{1}, 0}\right)+\frac{1}{2}\left(\mathbf{r}_{I, \Gamma_{2}}^{e, 0}-\mathbf{r}_{I, \Gamma_{2}}^{e_{2}, 0}\right)+\frac{1}{2}\left(\sigma_{I}^{e}-\sigma_{I}^{e_{1}}+\sigma_{I}^{e}-\sigma_{I}^{e_{2}}\right)
$$

By exploiting the above results, the local system for the patch of four elements can be rewritten as

$$
\left[\begin{array}{c}
\tilde{\mathbf{r}}_{I}^{e_{1}} \\
\tilde{\mathbf{r}}_{I}^{e_{2}} \\
\tilde{\mathbf{r}}_{I}^{e_{3}} \\
\tilde{\mathbf{r}}_{I}^{e_{4}}
\end{array}\right]=\frac{1}{2}\left[\begin{array}{cccc}
2 & -1 & 0 & -1 \\
-1 & 2 & -1 & 0 \\
0 & -1 & 2 & -1 \\
-1 & 0 & -1 & 2
\end{array}\right]\left[\begin{array}{c}
\sigma_{I}^{e 1} \\
\sigma_{I}^{e 2} \\
\sigma_{I}^{e 3} \\
\sigma_{I}^{e 4}
\end{array}\right]
$$

where we introduced notation

$$
\tilde{\mathbf{r}}_{I}^{e}=\mathbf{r}_{I}^{e}-\sum_{\Gamma \in \partial \Omega e, I}<\mathbf{r}_{I, \Gamma}^{e, 0}>; \quad<\mathbf{r}_{I, \Gamma}^{e, 0}>=\frac{1}{2}\left(\mathbf{r}_{I, \Gamma}^{e, 0}-\mathbf{r}_{I, \Gamma}^{e^{\prime}, 0}\right)
$$

The notation $\langle\cdot\rangle$ implies averaging. The $\left\langle\mathbf{r}_{I, \Gamma}^{e, 0}\right\rangle$ thus represent averaged boundary traction on $\Gamma$ (evaluated from $\mathrm{FE}$ solution) 'projected' to node $I$.

If we want to summarize, the element-wise boundary traction are computed from FE solution (based on chosen mathematical model) in two steps: (i) by solutions of patch-wise problems in (3.14) and (3.13) to obtain $\mathbf{r}_{I, \Gamma}^{e}$; (ii) by solutions of element-wise problems (3.9) to compute nodal values $p_{k}^{I}$ of boundary tractions (3.7).

\subsection{Equilibrated stress resultants for the DKQ element}

In order to find equilibrated stress resultants for the DKQ element, we start with interpolations for displacement and rotations. The variation of displacement along the edge $\Gamma$, which spans between element nodes $I$ and $J$, is 
defined by:

$$
w_{\Gamma}^{h}=w_{I} \varphi_{1}+w_{J} \varphi_{2}+\frac{l_{I J}}{4} \mathbf{n}_{I J} \cdot\left(\boldsymbol{\theta}_{I}-\boldsymbol{\theta}_{J}\right) \varphi_{3}+\frac{l_{I J}}{3} \Delta \theta_{I J} \varphi_{4}
$$

where parameter $\Delta \theta_{I J}$ can be expressed in terms of nodal displacements and rotations of neighboring nodes as $\Delta \theta=\frac{3}{2 l_{I J}}\left(w_{J}-w_{I}\right)-\frac{3}{4} \mathbf{n}_{I J} \cdot\left(\boldsymbol{\theta}_{I}+\boldsymbol{\theta}_{J}\right)$. Variation of rotations vector along the same edge can then be written as

$$
\boldsymbol{\theta}_{\Gamma}^{h}=\boldsymbol{\theta}_{I} \varphi_{1}+\boldsymbol{\theta}_{J} \varphi_{2}+\mathbf{n}_{I J} \Delta \theta_{I J} \varphi_{3}
$$

The functions $\varphi_{i}$ are

$$
\begin{aligned}
\varphi_{1} & =(1-\xi) / 2 \\
\varphi_{2} & =(1+\xi) / 2 \\
\varphi_{3} & =\left(\xi^{2}-1\right) / 2 \\
\varphi_{4} & =\xi\left(\xi^{2}-1\right) / 2
\end{aligned}
$$

In accordance with (3.15), (3.16) and notation used in (3.8), one can write the interpolation functions on the edge $\Gamma$ (between nodes $I$ and $J$ ) according to

$$
\begin{array}{ll}
N_{w, w}^{I}=\varphi_{1}-\varphi_{4} / 2 & N_{w, w}^{J}=\varphi_{2}+\varphi_{4} / 2 \\
N_{w, \theta_{x}}^{I}=-l n_{x}\left(-\varphi_{3}+\varphi_{4}\right) / 4 & N_{w, \theta_{x}}^{J}=-l n_{x}\left(+\varphi_{3}+\varphi_{4}\right) / 4 \\
N_{w, \theta_{y}}^{I}=-l n_{y}\left(-\varphi_{3}+\varphi_{4}\right) / 4 & N_{w, \theta_{y}}^{J}=-l n_{y}\left(+\varphi_{3}+\varphi_{4}\right) / 4 \\
N_{\theta_{x}, w}^{I}=-\left(3 n_{x} / 2 l\right) \varphi_{3} & N_{\theta_{x}, w}^{J}=+\left(3 n_{x} / 2 l\right) \varphi_{3} \\
N_{\theta_{x}, \theta_{x}}^{I}=\varphi_{1}-3 n_{x}^{2} \varphi_{3} / 4 & N_{\theta_{x}, \theta_{x}}^{J}=\varphi_{2}-3 n_{x}^{2} \varphi_{3} / 4 \\
N_{\theta_{x}, \theta_{y}}^{I}=-3 n_{x} n_{y} \varphi_{3} / 4 & N_{\theta_{x}, \theta_{y}}^{J}=-3 n_{x} n_{y} \varphi_{3} / 4 \\
N_{\theta_{y}, w}^{I}=-\left(3 n_{y} / 2 l\right) \varphi_{3} & N_{\theta_{y}, w}^{J}=+\left(3 n_{y} / 2 l\right) \varphi_{3} \\
N_{\theta_{y}, \theta_{x}}^{I}=-3 n_{x} n_{y} \varphi_{3} / 4 & N_{\theta_{y}, \theta_{x}}^{J}=-3 n_{x} n_{y} \varphi_{3} / 4 \\
N_{\theta_{y}, \theta_{y}}^{I}=\varphi_{1}-3 n_{y}^{2} \varphi_{3} / 4 & N_{\theta_{y}, \theta_{y}}^{J}=\varphi_{2}-3 n_{y}^{2} \varphi_{3} / 4
\end{array}
$$

where $l=l_{I J}, \mathbf{n}_{I J}=\left[n_{x}, n_{y}\right], \theta_{x}=\theta_{1}$ and $\theta_{y}=\theta_{2}$. Note, that with respect to (3.8) and notation in previous section one has: $k \in\left[w, \theta_{x}, \theta_{y}\right], m \in$ $\left[w, \theta_{x}, \theta_{y}\right],\left(\hat{v}_{\Gamma}^{h}\right)_{w}=\hat{w}_{\Gamma}^{h},\left(\hat{v}_{\Gamma}^{h}\right)_{\theta_{x}}=\hat{\theta}_{1 \Gamma}^{h},\left(\hat{v}_{\Gamma}^{h}\right)_{\theta_{y}}=\hat{\theta}_{2 \Gamma}^{h}$. 
The boundary stress resultants for the DKQ plate element at the edge $\Gamma$ will have three components

$$
\mathbf{t}=\left[q, m_{x}, m_{y}\right]
$$

where $q$ is the shear force along the edge defined in direction of $x=x_{3}$, and $m_{x}, m_{y}$ are the moments defined in the direction of $x=x_{1}$ and $y=x_{2}$, respectively. We choose to parameterize them as

$$
\begin{gathered}
q=q_{I} \varphi_{1}+q_{J} \varphi_{2} \\
m_{x}=m_{x I} \varphi_{1}+m_{x J} \varphi_{2} \\
m_{y}=m_{y I} \varphi_{1}+m_{y J} \varphi_{2}
\end{gathered}
$$

With respect to the notation introduced in (3.7), we note that

$$
\psi_{k}^{I}=\varphi_{1} \quad \psi_{k}^{J}=\varphi_{2}
$$

and

$$
\begin{aligned}
p_{q}^{I} & =q_{I} & p_{q}^{J} & =q_{J} \\
p_{m_{x}}^{I} & =m_{x I} & p_{m_{x}}^{J} & =m_{x J} \\
p_{m_{y}}^{I} & =m_{y I} & p_{m_{x}}^{J} & =m_{y J}
\end{aligned}
$$

To get the nodal parameters of (3.22) from (3.9) we have to evaluate integrals $\boldsymbol{M}_{\Gamma, n m}^{I J}=\int_{\Gamma} N_{m, n}^{I} \psi_{m}^{J} d s$, which have explicit forms as

$$
\boldsymbol{M}_{\Gamma, n m}^{I J}=\frac{l}{2} \int_{-1}^{+1}\left(\begin{array}{ccc}
N_{w, w}^{I} \psi_{w}^{J} & N_{\theta_{x}, w}^{I} \psi_{\theta_{x}}^{J} & N_{\theta_{y}, w}^{I} \psi_{\theta_{y}}^{J} \\
N_{w, \theta_{x}}^{I} \psi_{w}^{J} & N_{\theta_{x}, \theta_{x}}^{I} \psi_{\theta_{x}}^{J} & N_{\theta_{y}, \theta_{x}}^{I} \psi_{\theta_{y}}^{J} \\
N_{w, \theta_{y}}^{I} \psi_{w}^{J} & N_{\theta_{x}, \theta_{y}}^{I} \psi_{\theta_{x}}^{J} & N_{\theta_{y}, \theta_{y}}^{I} \psi_{\theta_{y}}^{J}
\end{array}\right) d \xi
$$

Remark 1: The so-obtained DKQ boundary stress resultants are exact replacement of the nodal residuals of the DKQ solution. If used for elementwise computation with the DKQ elements, they should produce exactly the same results as the original global computation. This fact can be used to check correctness of the boundary stress resultant computation.

Remark 2: Equilibrated stress resultants for the RMQ element can be obtained in a very similar way as for the DKQ element. Functions $N_{m, n}^{I}$ need to be found in accordance with the interpolations introduced in the previous section and used in (3.24). 


\subsection{Model error indicator for the DKQ element}

Model error indicator for the DKQ element is obtained by comparing its results with the ones obtained by an element based on more refined plate model (e.g. RMQ). As shown above, the later results can be obtained by element-wise computation with the DKQ boundary stress resultants applied as external edge loading. Thus, a Neumann-type problem has to be solved for each element of the mesh.

An element with only Neumman boundary conditions is essentially a floating structure, and one thus ought to eliminate the rigid body modes to get a unique result. This can be done simply by using the element geometry, as shown in [24]. A rigid body displacement $\mathbf{d}_{R}^{e}$ of an element is a linear combination of all its rigid body modes

$$
\mathbf{d}_{R}^{e}=\mathbf{D}^{\mathbf{e}} \boldsymbol{\alpha}
$$

where $\boldsymbol{\alpha}$ is a vector of amplitudes that correspond to rigid body modes, and $\mathbf{D}^{\mathbf{e}}$ is a matrix containing the rigid body modes of the element (arranged column-wise). Since the element stiffness matrix $\mathbf{K}^{\mathbf{e}}$ is singular due to the rigid body modes contribution, one can form a modified nonsingular stiffness matrix $\mathbf{K}^{\mathbf{e}^{\prime}}$ by adding a product $\mathbf{D}^{\mathbf{e}} \mathbf{D}^{\mathbf{e} T}$ to $\mathbf{K}^{\mathbf{e}}$

$$
\mathbf{K}^{\mathbf{e}^{\prime}}=\mathbf{K}^{\mathbf{e}}+\mathbf{D}^{\mathbf{e}} \mathbf{D}^{\mathbf{e} T}
$$

The inversion of $\mathbf{K}^{\mathbf{e}^{\prime}}$ is possible and solution $\mathbf{d}^{e^{\prime}}$ ('rigid-body-polluted' element nodal displacements/rotations) can be obtained. Since the matrix $\mathbf{D}^{\mathbf{e}} \mathbf{D}^{\mathbf{e} T}$ projects onto the space spanned by rigid body modes, we have

$$
\mathbf{d}^{e \prime}=\mathbf{d}^{e}+\mathbf{d}_{R}^{e}
$$

where $\mathbf{d}^{e}$ are real nodal displacements/rotations. As we are interested only in stress resultants, such a 'pollution' is not critical due to the following property $\mathbf{K}^{e} \mathbf{d}_{R}^{e}=\mathbf{0}$.

Having defined the stress resultants for the RMQ, the RMQ deformation energy can be calculated, by taking into account the corresponding bending and shear deformations

$$
\begin{aligned}
E^{R M,{ }^{e}} & =\int_{\Omega}^{e} \mathbf{m}^{R M, T} \mathbf{C}^{B^{-1}} \mathbf{m}^{R M} d \Omega+\int_{\Omega}^{e} \mathbf{q}^{R M,{ }^{T}} \mathbf{C}^{S^{-1}} \mathbf{q}^{R M} d \Omega \\
& =E_{B}^{R M, e}+E_{S}^{R M, e}
\end{aligned}
$$


where $\mathbf{m}^{R M}=\left[m_{11}, m_{22}, m_{12}\right]^{T}$ and $\mathbf{q}^{R M}=\left[q_{1}, q_{2}\right]^{T}$.

Since DKQ and RMQ plate elements predict very similar values for moments and the DKQ assumes zero shear strains, the shear deformation en$\operatorname{ergy} E_{S}^{R M, e}$ of RMQ can be used to determine how well the DKQ plate model performs in a given situation. If shear strain contribution is low enough, the DKQ could be considered as a sufficiently good model, otherwise it should be replaced by a more suitable element based on Reissner-Mindlin model (i.e. by the RMQ). A fairly reasonable choice for a model error indicator $\eta_{e}^{M}$ we use herein is therefore the ratio of the energy norm of the shear difference between the RMQ and the DKQ element to the total energy of the RMQ element. Due to the zero shear strain assumption in the DKQ, and similar prediction for moments of both elements, one can define the model error indicator as

$$
\eta_{e}^{M}=E_{S}^{R M, e} / E^{R M,^{e}}
$$

This indicator is used below in all numerical examples.

\section{$4 \quad$ Numerical examples}

We present in this section results of numerical examples, which were chosen to illustrate performance of the proposed approach to adaptive modelling of plates. We do not elaborate on the mesh adaptivity for controlling the discretization error, since this has been extensively covered in the literature (e.g., see [22], [17], [29], [10]). However, the departure point for the present model adaptivity procedure should be an optimally adapted FE mesh, with the discretization error uniformly distributed and placed within a predefined limit. We note that the plate elements in such an automatically generated mesh of quadrilaterals, which takes into account predefined element size distribution, can be quite distorted. For this reason we also present examples where the influence of mesh distortion on the chosen model error indicator is studied. 


\subsection{Sensitivity of model error indicator on mesh dis- tortion}

\subsubsection{Simply supported square plate}

Problem of simply supported (SSSS) square plate under uniform loading is used to test the sensitivity of model error indicator on mesh distortion; as well as to compare those effects for thin and thick plate situations. The material is linear elastic and isotropic, with Young's modulus $E=10.92$ and Poisson's ratio $\nu=0.3$. The side length is $a=10$. Two values for the plate thickness $t=0.1$ (thin) and $t=1$ (thick) are selected. Due to the symmetry, only one quarter of the plate (the lower left one) is chosen for the numerical analysis.

The model error indicator (3.27) is computed for each DKQ element of the mesh. This value is then compared with the corresponding analytical (reference) value, which can be for this simple problem obtained by using analytical (series) solution for Mindlin plates, see e.g. [19], [8]. In Figure 3, we show the comparison of two structured (regular) meshes with the same number of elements for the case of thick plate. The meshes differ in discretization error, which is lower for the second mesh that is more refined towards the outer edges. It can be observed that the computed model error indicator is very close to the reference one, except for the corner element. This is due to the fact that the equilibration is not possible to carry out at the corner node. We thus obtain as a consequence that the estimated boundary stress resultants for the corner element are not as good as for the inner elements.

To estimate the effect of mesh distortion, the model error indicator (3.27) is computed (for the case of thick plate) with two distorted meshes. The first mesh is obtained by adaptive mesh refinement with $5 \%$ tolerance in discretization error. The second one is derived from the mesh shown in Figure 3 by a parametrical displacement of the nodes. The results are presented in Figure 4. Again, comparison is made with the analytical (reference) solution. We note that the performance of model error indicator is slightly better for parametrical distortion of the mesh. One may conclude from the results shown in Figure 4 that the model error indicator is slightly (but not severely) influenced by mesh distortion.

We repeat the above mentioned comparison also for the case of thin plate. The result are shown in Figure 5 and 6 (notice the change of scale). The 
model error indicator shows that the DKQ is sufficiently good (almost) for the whole mesh. Comparison with the analytical solution shows that the sensitivity of model error indicator is of the same range as in the case of thick plate.

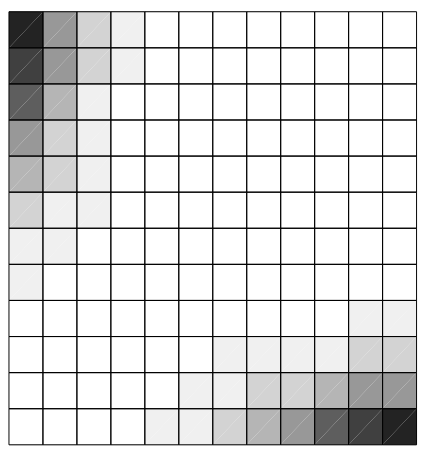

(a)

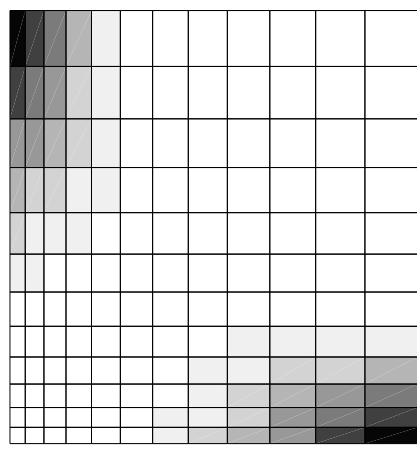

(a)

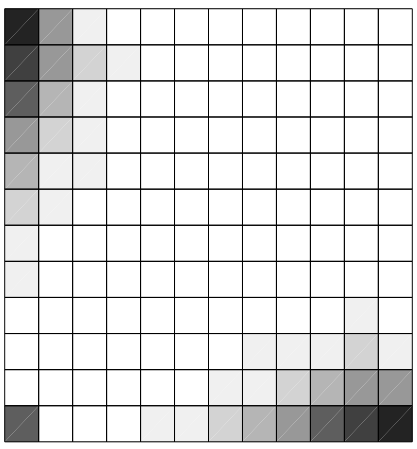

(b)

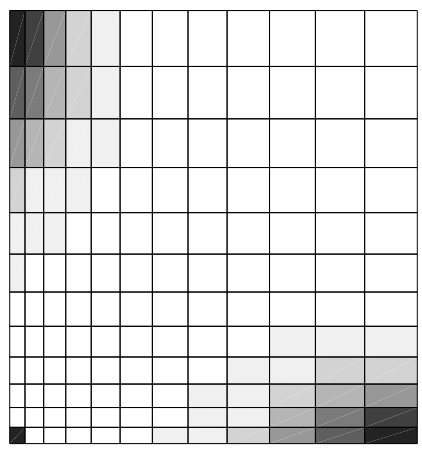

(b)

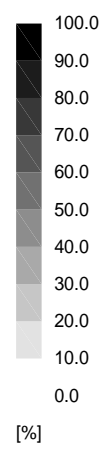

[\%]

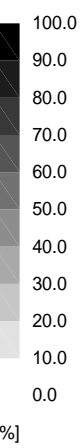

[\%]

Figure 3: Reference value of model error indicator (a) and its estimated value (b) for thick SSSS plate for rectangular meshes

\subsubsection{Square plate with two simply supported and two free edges}

The second problem we study is a square plate with two opposite edges simply supported and two other edges free (SFSF). It has been chosen to test the ability of the proposed procedure to detect boundary layer effect, which is most prominent along the free edges, see [2]. The width of the 


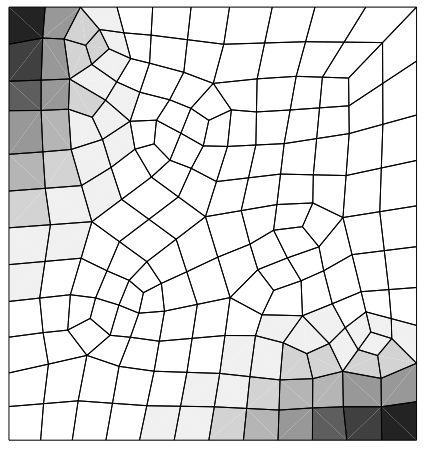

(a)

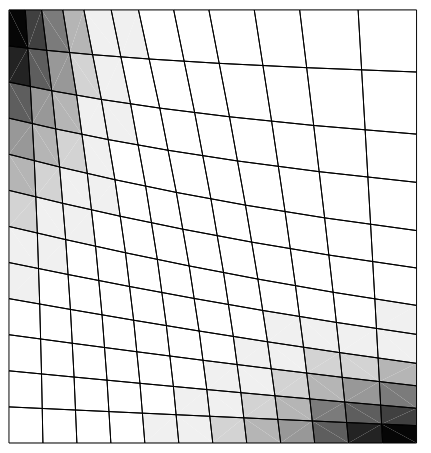

(a)

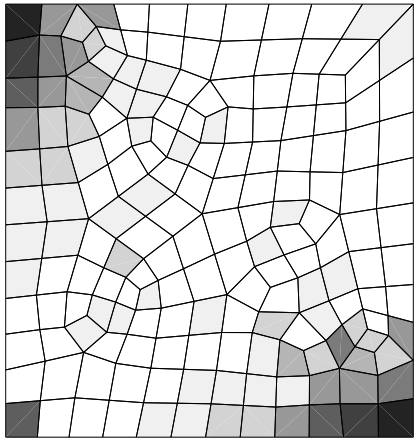

(b)

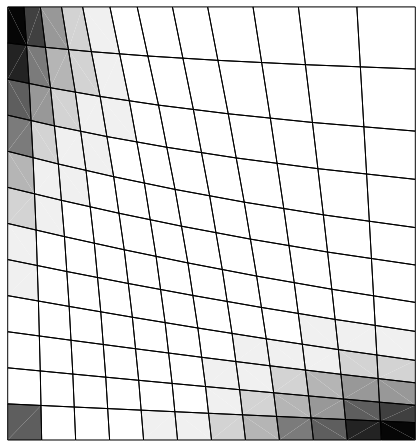

(b)

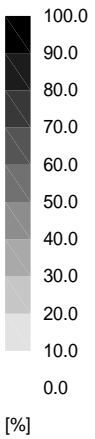

[\%]

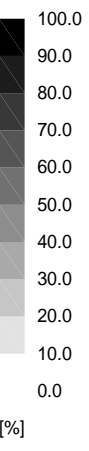

[\%]

Figure 4: Reference value of model error indicator (a) and its estimated value (b) for thick SSSS plate for distorted meshes

boundary layer is proportional to the thickness of the plate, and it is most pronounced for the transverse shear component, which varies as $1 / t$. The geometry and material parameters are the same as in the first example. Due to the symmetry, only one quarter of the plate (the lower left one) is used in the analysis; the boundary conditions are imposed as follows: the left edge in simply supported, the lower one is free, and the right and the upper ones take into account symmetry.

Since the boundary layer effect is intrinsic to the Reissner-Mindlin theory, the DKQ can not detect it. In the present procedure the equilibrated boundary stress resultants are derived from the DKQ solution and subsequently applied to the RMQ in order to evaluate model error indicator. It is 


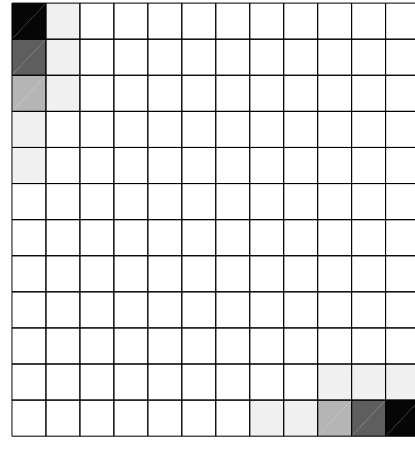

(a)

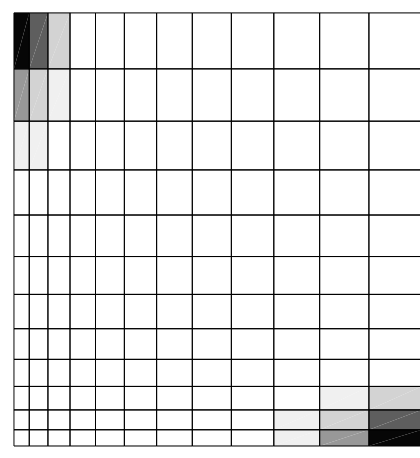

(a)

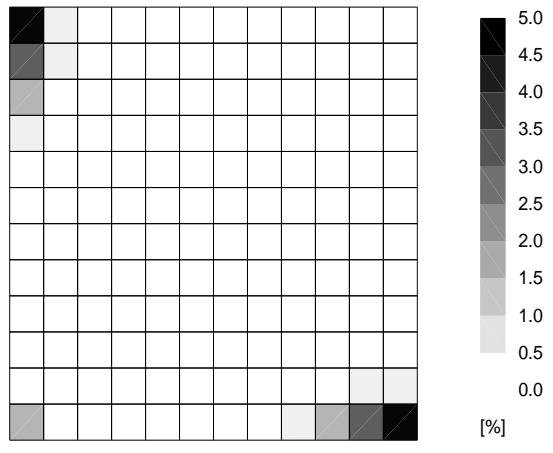

(b)

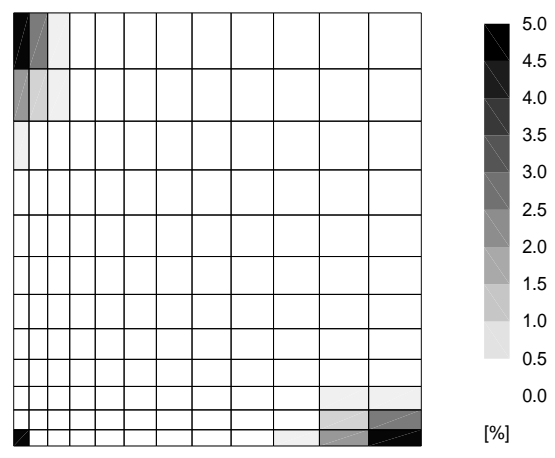

(b)

Figure 5: Reference value of model error indicator (a) and its estimated value (b) for thin SSSS plate for rectangular meshes

therefore interesting to see weather this procedure can effectively detect the boundary layer. The result shown in Figure 7 indicates that the procedure is able to detect a region with increased shear deformation energy (along the left edge) but is not very successful to detect the boundary layer (along the lower free edge), which is clearly observable in the reference (analytical) solution. Comparison with the analytical solution shows (see Figures 7, 8, 9 and 10) that the sensitivity of model error indicator is of the same range as in the case of SSSS plate. 


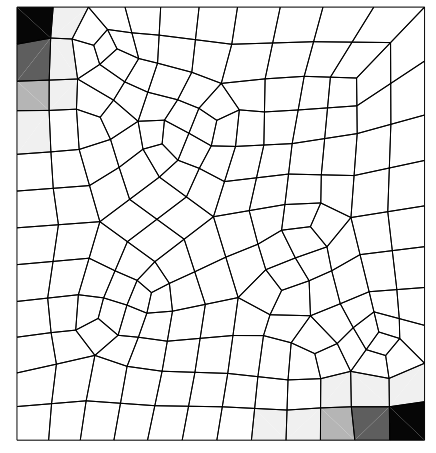

(a)

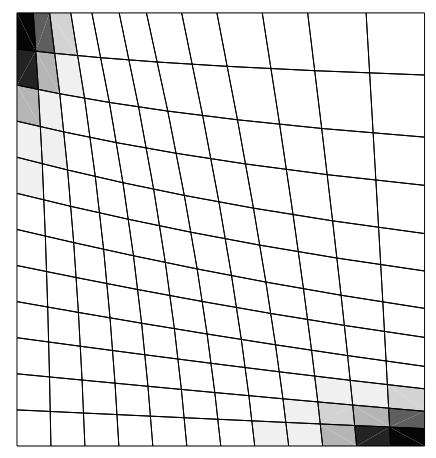

(a)

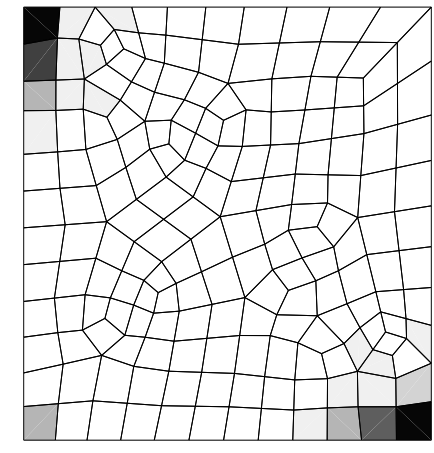

(b)

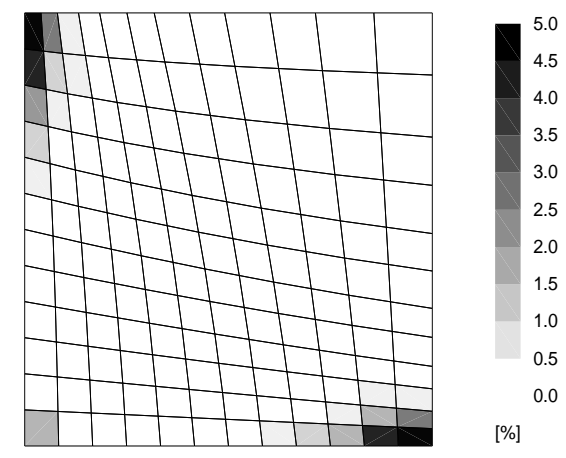

(b)

Figure 6: Reference value of model error indicator (a) and its estimated value (b) for thin SSSS plate for distorted meshes

\subsection{Adaptive analysis of L-shaped plate}

Adaptive model analysis of an L-shaped plate under uniform unit pressure is considered in this example. Long sides $(a=10)$ of the plate are clamped, all other sides $(b=5)$ are free. The plate is made of linear elastic isotropic material, with Young's modulus $E=10.92$ and Poisson's ratio $\nu=0.3$. Thickness of the plate is equal to 1 . Non distorted mesh was chosen for this example in order to avoid effect of element distortion on model error indicator.

The computation started with the analysis of the plate by using DKQ elements. The model error indicator (3.27) was further computed and the 


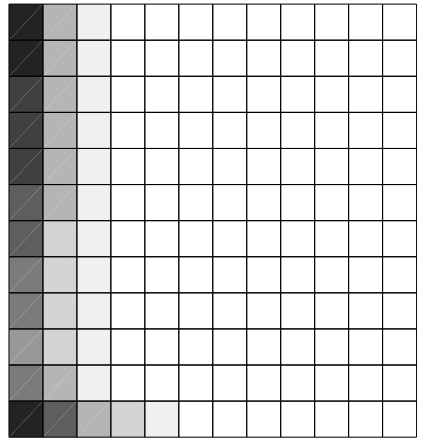

(a)

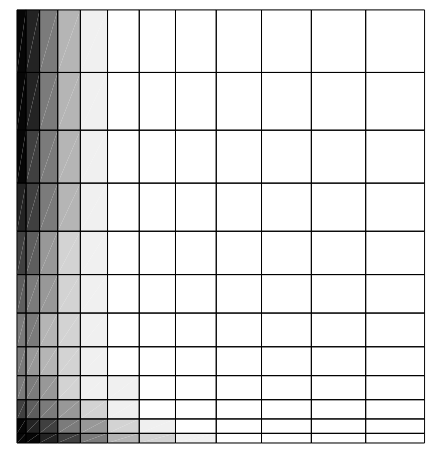

(a)

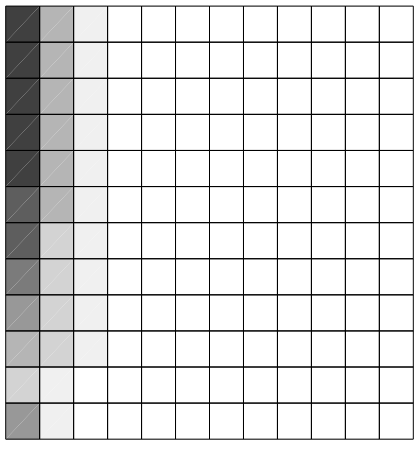

(b)

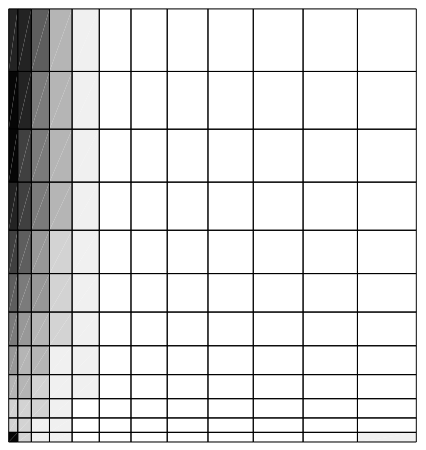

(b)

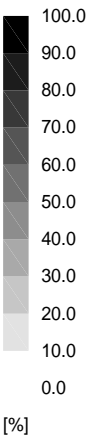

[\%]

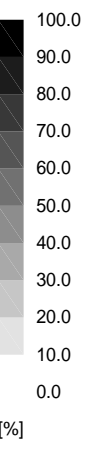

[\%]

Figure 7: Reference value of model error indicator (a) and its estimated value (b) for thick SFSF plate for rectangular meshes

result is shown in Figure 11. The indicator identified several regions where the model error was high. The limit of $15 \%$ model error was chosen to determine the elements of the mesh, where the RMQ should be used instead of the DKQ. Those elements are colored dark grey in Figures 12-14 (b). Finally, the computation with both the DKQ (light grey in Figures 12-14 (b)) and the RMQ elements (dark grey in Figures 12-14 (b)), used at different regions of the plate, was performed. Kinematic coupling of elements based on different models was considered, i.e. the hierarchic rotation $\Delta \theta$ of the RMQ was restrained on edges where the RMQ elements were adjacent to the DKQ elements in order to account for zero transverse shear modelling along those edges. 


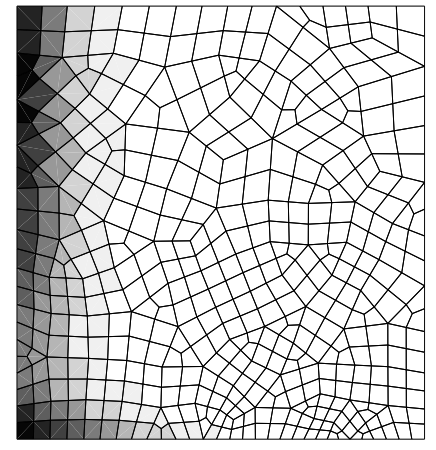

(a)

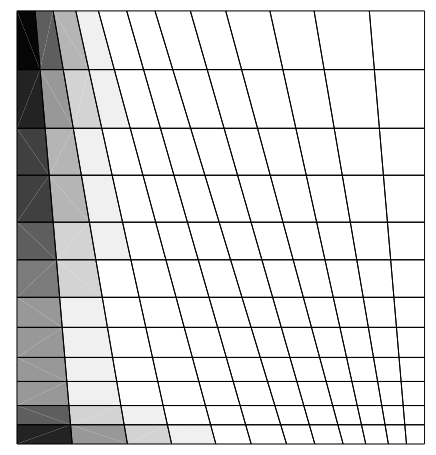

(a)

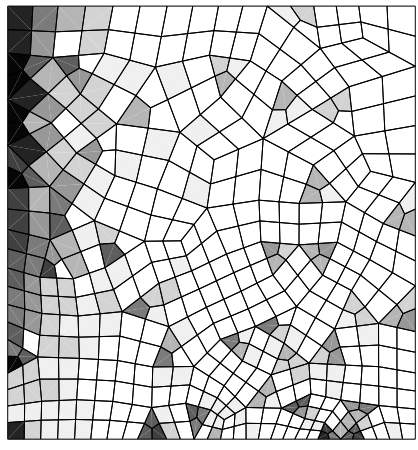

(b)

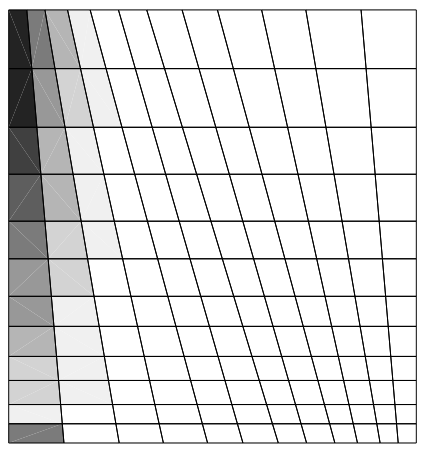

(b)

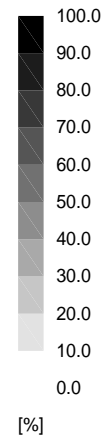

[\%]

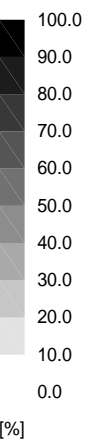

[\%]

Figure 8: Reference value of model error indicator (a) and its estimated value (b) for thick SFSF plate for distorted meshes

The comparison of stress resultants evaluated with the DKQ elements (Figures 12-14 (a)), with the RMQ elements (Figures 12-14 (c)) and with both the DKQ and the RMQ elements (Figures 12-14 (d)) is shown. It can be seen that the RMQ based computation and the mixed DKQ-RMQ based computation produce almost identical results; on the other hand, the results are not equal to those obtained by using only DKQ elements. Therefore, the comparison of these different results (and especially close agreement between the DKQ-RMQ and the RMQ computations), clearly indicates that the adaptive procedure of this kind performs successfully. One can hope for the same trend when applying the same procedure to higher order plate models, where a significant computational savings could also be obtained in 


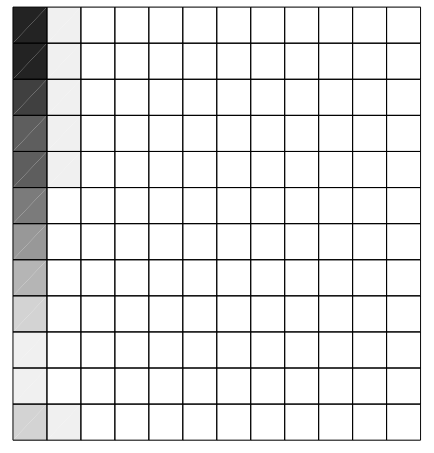

(a)

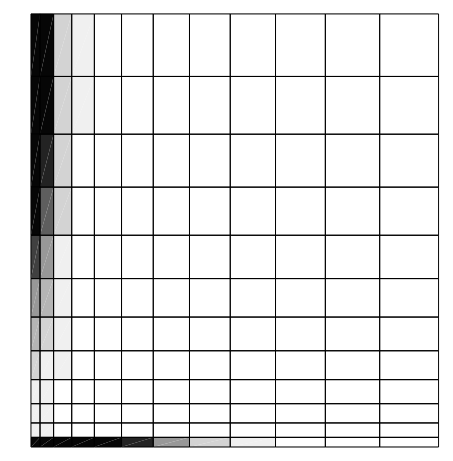

(a)

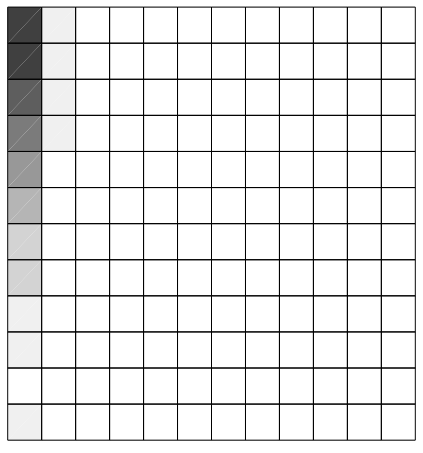

(b)

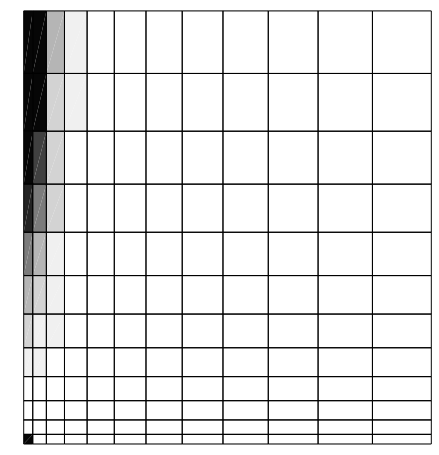

(b)

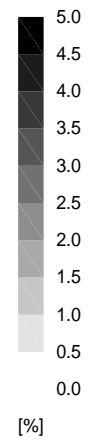

$[\%]$

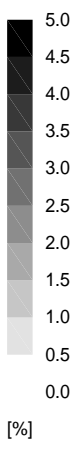

$[\%]$

Figure 9: Reference value of model error indicator (a) and its estimated value (b) for thin SFSF plate for rectangular meshes

the process.

\subsection{Adaptive analysis of Morley's skew plate}

Adaptive model analysis of Morley's $30^{\circ}$ skew plate (see [20]) with thickness $t=1$, side length $a=10$, simple supports on all sides, and unit uniform pressure is performed in this example. The plate is built of linear elastic isotropic material, with Young's modulus $E=10.92$ and Poisson's ratio $\nu=0.3$. The most interesting feature of the solution to this problem concerns two singular points at the two obtuse corners of the plate, which strongly influence the quality of the computed results (e.g. see [13]). The chosen FE 


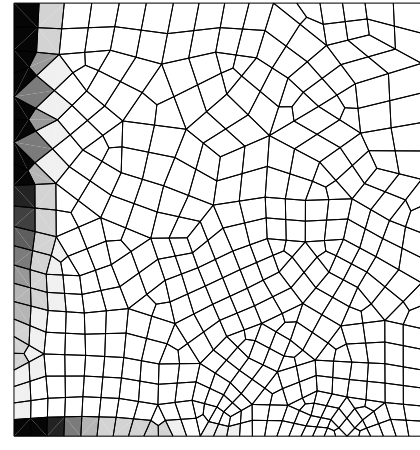

(a)

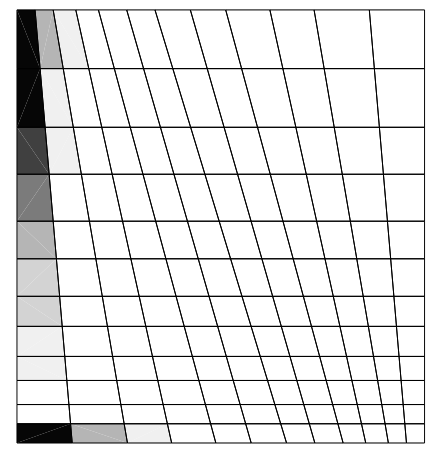

(a)

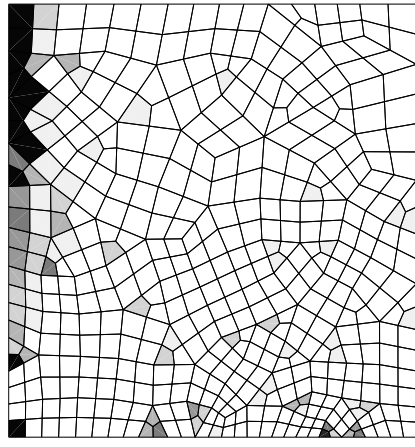

(b)

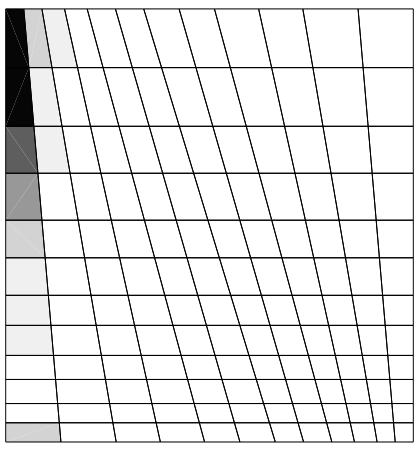

(b)

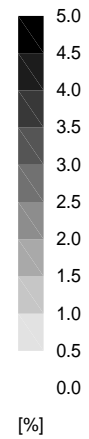

[\%]

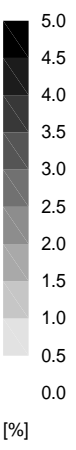

[\%]

Figure 10: Reference value of model error indicator (a) and its estimated value (b) for thin SFSF plate for distorted meshes

mesh is made more dense near the sides of the plate where singularities are expected.

The computation started with the analysis by using DKQ elements. The model error indicator (3.27) was further computed, see Figure 15. It can be seen from Figure 15 that the error was increased near the sides and in the vicinity of the obtuse corners. Again, the limit of $15 \%$ model error was chosen to determine the elements of the mesh, where the RMQ should be used instead of the DKQ; all such elements are colored dark grey in (b) part of Figures 16 and 17. Finally, the computation with both the DKQ (light grey in Figures 16(b) and 17(b)) and the RMQ elements (dark grey in Figures 16-17 (b)), used at different regions of the plate, was performed. 

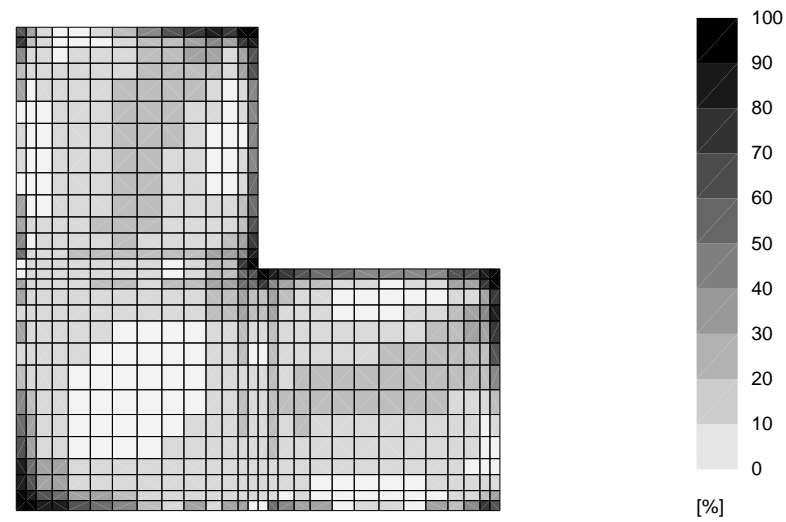

Figure 11: Model error indicator

It can be seen in Figure 16 that in this case the difference in results for moments between all three computational models is negligible. The difference however still shows in the results for the shear force, which are presented in Figure 18.

\section{Conclusions}

We have presented a detailed development for plate model adaptivity procedure capable of selecting automatically the best suitable plate finite element between the two plate models, the first for thin Kirchhoff plate and the second for thick Reissner-Mindlin plate. The model adaptivity computation is carried out on the element basis, and it starts with the finite element mesh which is first passed through the standard mesh adaptivity procedure in order to achieve the acceptable discretization errors throughout the mesh. We addressed in detail the particular case of two low-order plate finite element models, represented with discrete Kirchhoff quadrilateral (DKQ) and Reissner-Mindlin quadrilateral (RMQ); but the same procedure would carry over to higher-order plate models that capture more accurately local 3D effects.

It is the key advantage of the presented approach to plate model adap- 


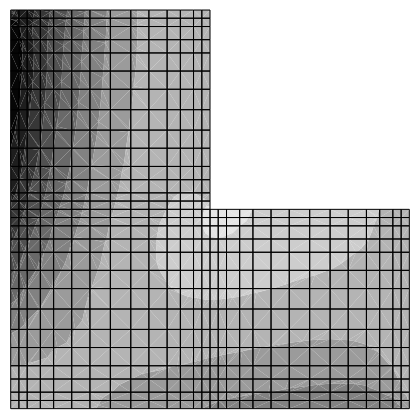

(a)

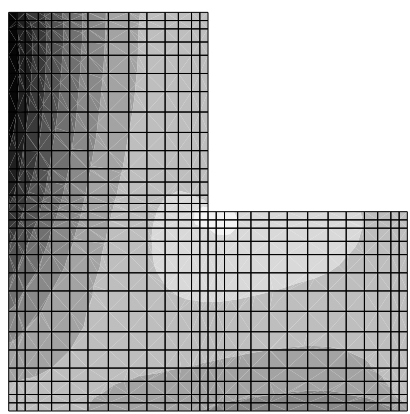

(c)

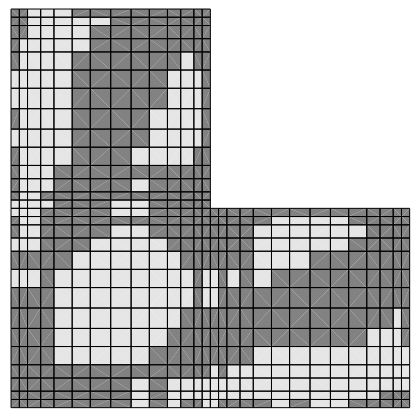

(b)

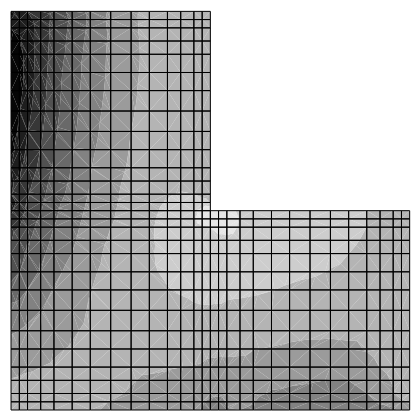

(d)
11.70

10.16

8.61

7.07

5.52

3.98

2.43

0.89

$-0.66$

$-2.20$

$-3.75$

Figure 12: Stress resultant $m_{x x}$ (a) DKQ, (c) RMQ and (d) DKQ-RMQ model. The modelisation of (d) is presented in (b), where dark areas represent RMQ and light ones DKQ model.

tivity to remain uncoupled with respect to the mesh adaptivity procedure employed to reduce the discretization error, and thus can be combined with any already available mesh adaptivity procedure to ensure the optimal overall accuracy. This advantage stems from the proposed special choice of the finite element interpolations for both RMQ and DKQ plate elements, which results with the same quality of the discrete approximation for bending moments.

Numerical examples illustrate clearly that the proposed procedure is capable of capturing any significant contribution of shear deformations. The case in point concerns the shear layers which typically occur for different kinds of boundary conditions. 


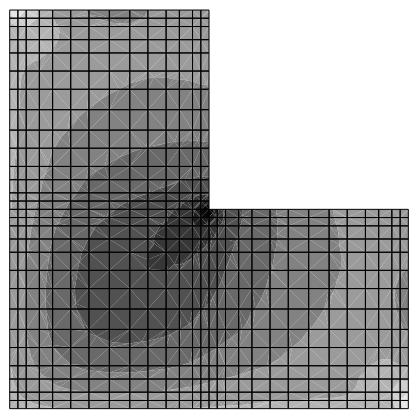

(a)

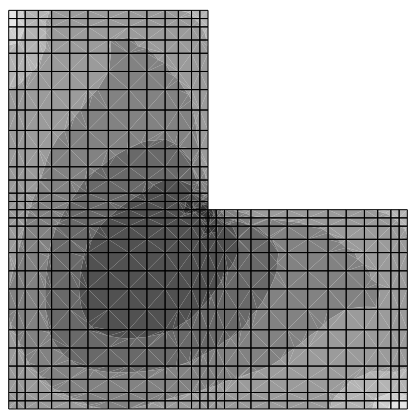

(c)

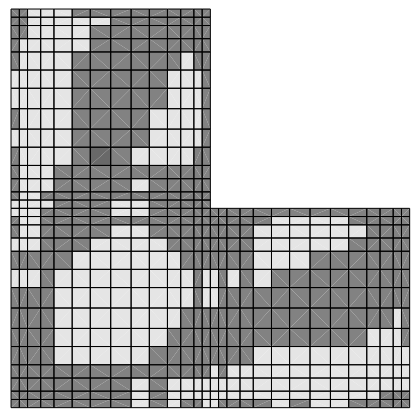

(b)

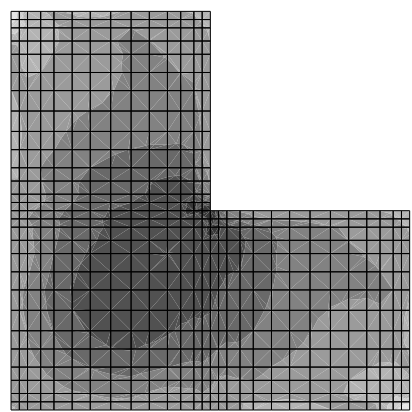

(d)
131.520

3.03

2.55

2.06

1.57

1.08

0.60

0.11

$-0.38$

$-0.87$

$-1.36$

Figure 13: Stress resultant $m_{x y}$ (a) DKQ, (c) RMQ and (d) DKQ-RMQ model. The modelisation of (d) is presented in (b), where dark areas represent RMQ and light ones DKQ model.

Acknowledgements: The work described herein is funded by the French Ministry of Research and French-Slovenian collaboration program "Proteus". The Slovenian Research Agency Award for foreign scientists with recognized qualifications to Adnan Ibrahimbegovic is also gratefully acknowledged.

\section{References}

[1] M. Ainsworth and J. T. Oden. A posteriori error estimation in finite element analysis. Wiley Inter-Science, 2000. 


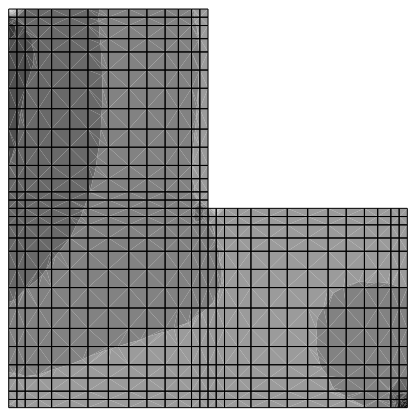

(a)

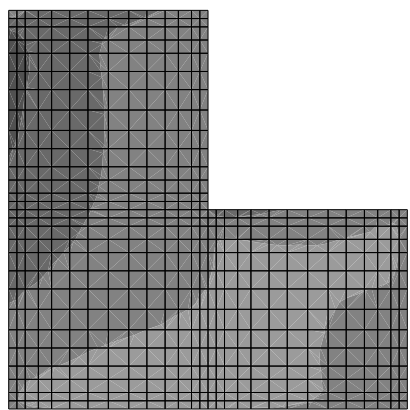

(c)

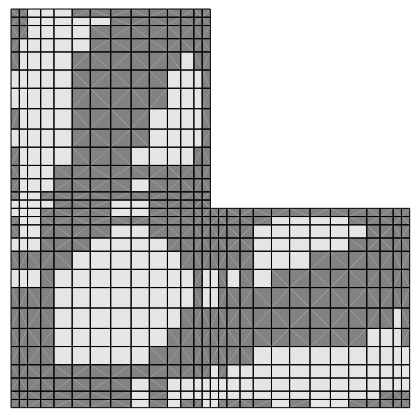

(b)

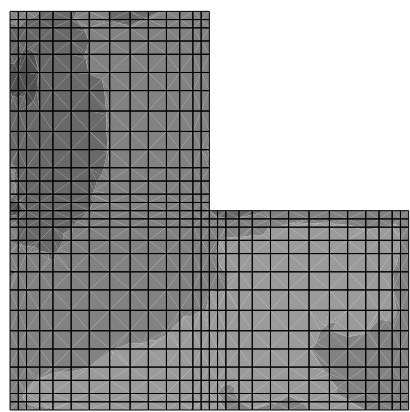

(d)

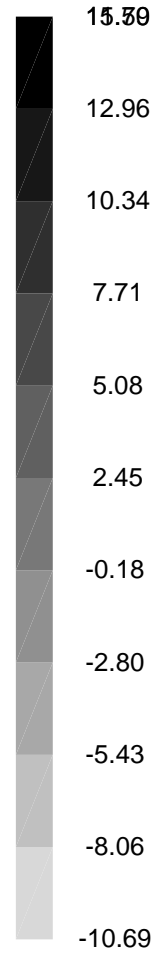

$-10.69$

Figure 14: Stress resultant $g_{x}$ (a) DKQ, (c) RMQ and (d) DKQ-RMQ model. The modelisation of $(\mathrm{d})$ is presented in $(\mathrm{b})$, where dark areas represent RMQ and light ones DKQ model.

[2] D. N. Arnold and R. S. Falk. Edge effects in the reissner-mindlin plate theory. Proc. Symp. Analyt. Comput. Models Shells, San Francisco, ASME Winter Annual Meeting, 1989.

[3] I. Babuška and L. Li. The problem of plate modeling. theoretical and computational results. Computer Methods in Applied Mechanics and Engineering, 100:249-273, 1992.

[4] I. Babuška and C. Schwab. A posteriori error estimation for hierarchic models of elliptic boundary value problem on thin domains. Technical 


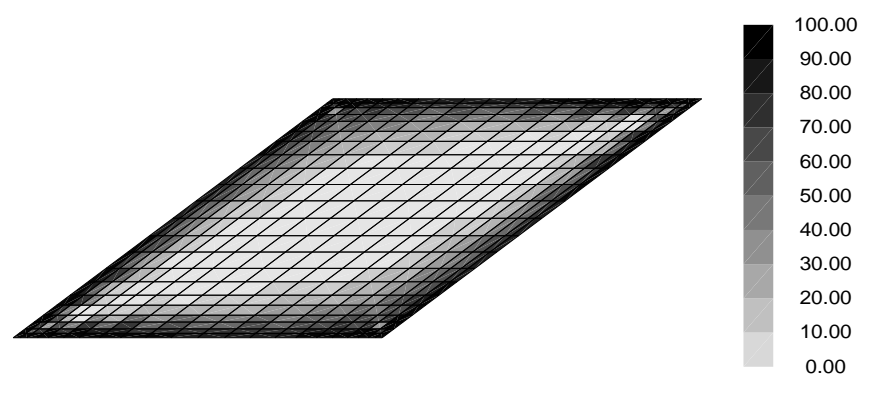

$[\%]$

Figure 15: Model error indicator

note BN-1148, Institute for Physical Science and Technology, University of Maryland, College Park, 1993.

[5] K.-J. Bathe. Finite element procedures. Prentice-Hall, 1996.

[6] K.-J. Bathe and E. Dvorkin. A four-node plate bending element based on mindlin-reissner plate theory and a mixed interpolation. Int. J. Numer. Methods Eng., 21:367-383, 1985.

[7] J.-L. Batoz and G. Dhatt. Modélisation par éléments finis en mécanique des structures, vol II : Plaques. Hermes Science-Lavoisier, 1990.

[8] B. Brank. On boundary layer in the mindlin plate model. levy plates. Thin-walled structures (submitted), 2007.

[9] B. Brank and E. Carrera. A family of shear-deformable shell finite elements for composite structures. Composite Structures, 76:287-297, 2000 .

[10] T. Grätsch and K.-J. Bathe. A posteriori error estimation techniques in practical finite element analysis. Computers and Structures, 83:235-265, 2005.

[11] E. Hinton and J. Campbel. Local and global smoothing of discontinuous finite element function using a least squares method. International Journal for Numerical Methods in Engineering, 8:461-480, 1974. 


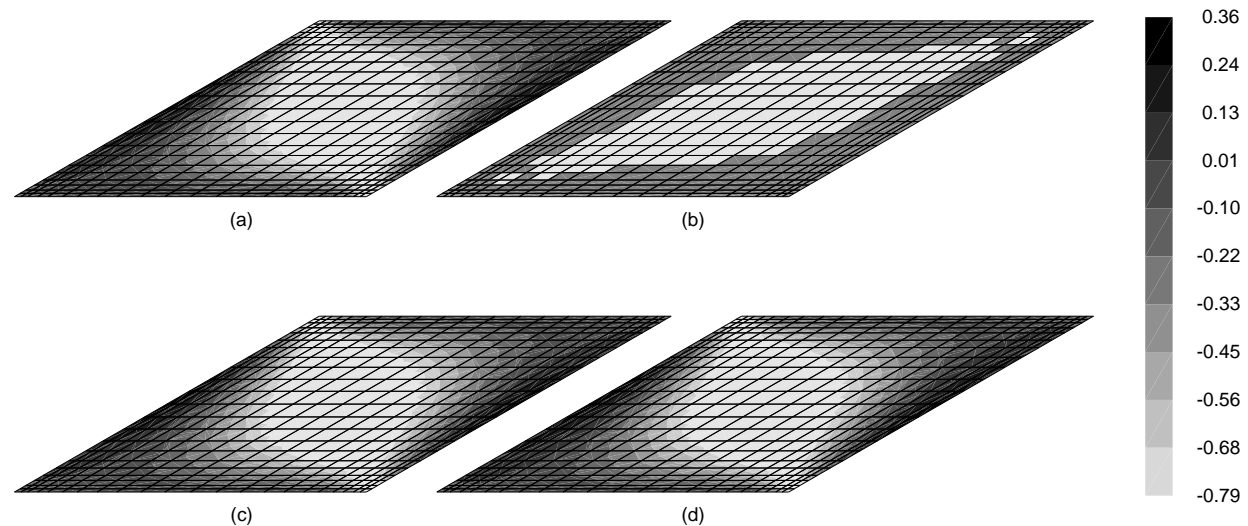

Figure 16: Stress resultant $m_{x x}$ computed with: (a) DKQ, (c) RMQ and (d) DKQ-RMQ model. The modelisation of $(d)$ is presented in (b), where dark areas represent RMQ and light ones DKQ model.

[12] A. Ibrahimbegovic. Plate quadrilateral finite element with incompatible modes. Communications in Applied Numerical Methods, 8:497-504, 1992 .

[13] A. Ibrahimbegovic. Quadrilateral finite elements for analysis of thick and thin plates. Computer Methods in Applied Mechanics and Engineering, 110:195-209, 1993.

[14] A. Ibrahimbegovic. Mechanique non lineare des solides deformables: formulation théorique et implantation éléments finis. Hermes Science Lavoisier, 2006.

[15] P. Ladevèze and D. Leguillon. Error estimate procedure in the finite element method and applications. SIAM Journal of Numerical Analysis, 20(3):485-509, 1983.

[16] P. Ladevèze and E. Maunder. A general method for recovering euilibrium element tractions. Computer Methods Mech. Eng., 137:111-151, 1996.

[17] P. Ladevèze and J. Pelle. Mastering calculation in linear and nonlinear mechanics. Springer, 2004. 


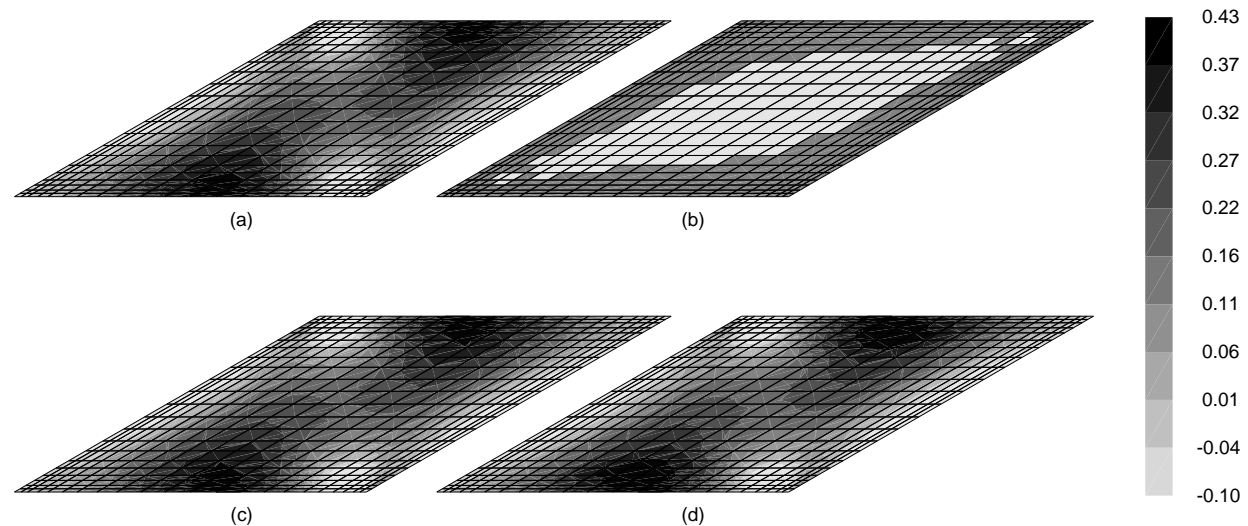

Figure 17: Stress resultant $m_{x y}$ computed with: (a) DKQ, (c) RMQ and (d) DKQ-RMQ model. The modelisation of $(d)$ is presented in (b), where dark areas represent RMQ and light ones DKQ model.

[18] C. K. Lee and R. E. Hobbs. Automatic adaptive refinement for plate bending problems using reissner-mindlin plate bending elements. International Journal for Numerical Methods in Engineering, 41:1-63, 1998.

[19] K. H. Lee, G. T. Lim, and C. M. Wang. Thick levy plates re-visited. International Journal of Solids and Structures, 39:127-144, 2002.

[20] L. Morley. Skew plates and structures. Pergamon Press - MacMillan Company, 1963.

[21] J. T. Oden and J. R. Cho. Local a posteriori error estimation of hierarchical models for plate- and shell-like structures. Computer in Mathematics with Applications, 149:33-48, 1997.

[22] J. T. Oden, S. Prudhomme, and D. C. Hammermand. Modeling error and adaptivity in nonlinear continuum mechanics. Computer Methods in Applied Mechanics and Engineering, 190:6663-6684, 2001.

[23] S. Ohnimus, E. Stein, and E. Walhorn. Local error estimates of fem for displacements and stresses in linear elasticity by solving local neumann problems. International Journal for Numerical Methods in Engineering, 52:727-746, 2001. 


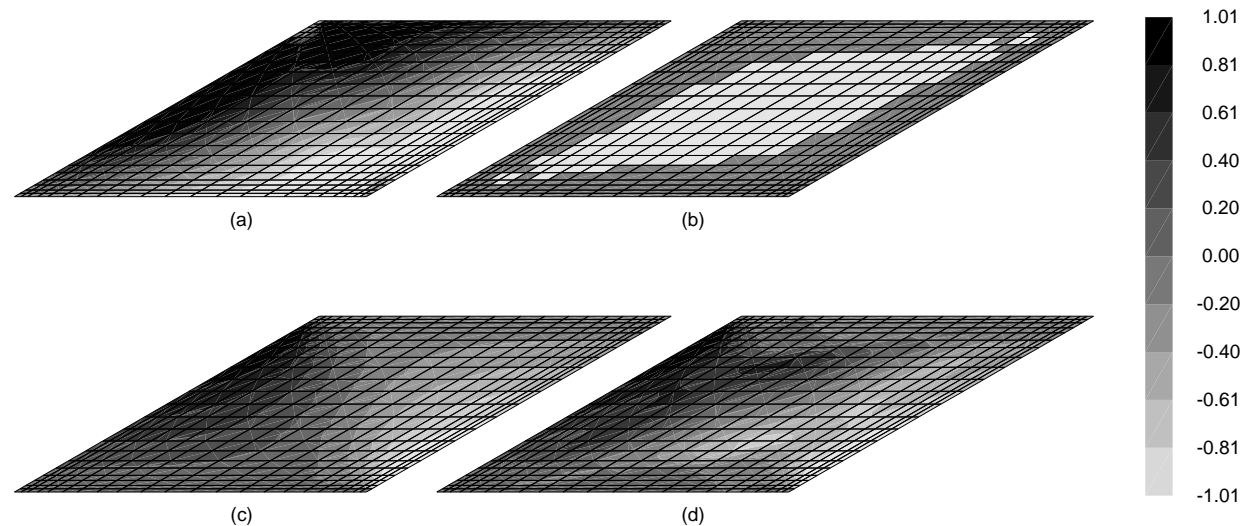

Figure 18: Stress resultant $q_{x}$ computed with: (a) DKQ, (c) RMQ and (d) DKQ-RMQ model. The modelisation of $(d)$ is presented in (b), where dark areas represent RMQ and light ones DKQ model.

[24] K. C. Park and C. A. Felippa. A variational framework for solution method developments in structural mechanics. Journal of Applied Mechanics, 65/1:242-249, 1998.

[25] E. Stein and S. Ohnimus. Coupled model- and solution adaptivity in the finite element method. Computer Methods in Applied Mechanics and Engineering, 150:327-350, 1997.

[26] E. Stein and S. Ohnimus. Anisotropic discretization- and model-error estimation in solid mechanics by local neumann problems. Computer Methods in Applied Mechanics and Engineering, 176:363-385, 1999.

[27] E. Stein, M. Rüter, and S. Ohnimus. Adaptive finite element analysis and modelling of solids and structures. findings, problems and trends. International Journal for Numerical Methods in Engineering, 60:103138, 2004.

[28] E. Wilson. SAP 2000, Analysis reference. Computers \& Structures Inc., 1998.

[29] O. C. Zienkiewicz and R. L. Taylor. Finite element method. Elseiver, 2000 . 
[30] O. C. Zienkiewicz and J. Z. Zhu. The superconvergent patch recovery and a posteriori error estimates. part 1: The recovery technique. International Journal of Numerical Methods in Engineering, 33:1331-1364, 1992. 